The Astronomical JouRnaL, 118:1373-1389, 1999 September

(C) 1999. The American Astronomical Society. All rights reserved. Printed in U.S.A.

\title{
RR LYRAE LUMINOSITY DIFFERENCES BETWEEN OOSTERHOFF GROUP I AND II CLUSTER SYSTEMS AND THE ORIGIN OF THE OOSTERHOFF DICHOTOMY
}

\author{
JAE-Woo Lee AND Bruce W. CARneY \\ Department of Physics and Astronomy, University of North Carolina, Chapel Hill, NC 27599-3255; jaewoo@astro.unc.edu, \\ bruce@physics.unc.edu \\ Received 1999 March 22; accepted 1999 May 27
}

\begin{abstract}
We present a comparative study of the Oosterhoff II cluster M2 and the Oosterhoff I cluster M3. Both have similar metallicities, $[\mathrm{Fe} / \mathrm{H}]=-1.62$ for $\mathrm{M} 2$ and -1.66 for $\mathrm{M} 3$, but very different horizontal-branch (HB) morphologies $(B-R) /(B+V+R)=0.92$ for M2 and 0.08 for M3. A period shift analysis and main-sequence fitting show that RRab variables in $\mathrm{M} 2$ are about 0.2 mag brighter than those in M3. Comparisons of the M2 period shift with Oosterhoff I clusters NGC 3201 and NGC 7006 also yield similar results, while a comparison between M2 and the Oosterhoff II cluster NGC 5986 reveals that the RR Lyrae luminosities are very similar. The luminosity difference is thought to be due to the evolutionary effect described in 1990 by Lee, Demarque, \& Zinn: the M2 RRab variables have evolved away from the zero-age horizontal branch (ZAHB), while most M3 RRab variables lie near the ZAHB. A comparison of the mean period change rates of two clusters supports this hypothesis. Our relative age estimation using the difference in color between the base of giant branch and turn-off point shows that M2 is about 2 Gyr older than M3. Our result strongly suggests that the Oosterhoff dichotomy is due to age differences between Oosterhoff group I and II. This is consistent with the idea that the global second parameter is age.

We discuss the kinematic differences between Oosterhoff group I and II clusters. Our result shows that the Oosterhoff group I clusters have zero or retrograde rotation with $\left\langle v_{\text {rot }}\right\rangle=-68 \pm 56 \mathrm{~km} \mathrm{~s}^{-1}$ and $\sigma_{\text {los }}=131 \pm 28 \mathrm{~km} \mathrm{~s}^{-1}$, while the Oosterhoff group II clusters have prograde rotation with $\left\langle v_{\text {rot }}\right\rangle=$ $+94 \pm 47 \mathrm{~km} \mathrm{~s}^{-1}$ and $\sigma_{\mathrm{los}}=115 \pm 29 \mathrm{~km} \mathrm{~s}^{-1}$, confirming a similar conclusion of van den Bergh. The difference in kinematics and ages between Oosterhoff group I and II clusters suggests that they may have different origins: The Oosterhoff II clusters were formed very early in the proto-Galaxy while the Oosterhoff I clusters were formed at different locations and at a later time, and were probably merger events. The period distributions of an unbiased sample of field RRab variables with $|Z| \leq 3 \mathrm{kpc}$ and $|Z| \geq 5$ $\mathrm{kpc}$ indicate that they may belong to different populations, with peak periods of 0.65 and 0.55 days, respectively. If the hypothesis that the Oosterhoff dichotomy is due to evolution is correct, then this period shift among the field RR Lyrae variables suggests that the RRab population with $|Z| \leq 3 \mathrm{kpc}$ is somewhat older than the RRab population with $|Z| \geq 5 \mathrm{kpc}$. This also suggests different formation histories.
\end{abstract}

In an appendix, we discuss that the frequently used Gaussian HB mass-dispersion rate (i.e., the massloss rate at the red giant branch [RGB] tip) in synthetic HB model calculations cannot fully explain the extended blue HB population and the pulsational properties of RR Lyrae variables in M2. Comparisons with synthetic HB models strongly suggests that an enhanced mass loss is required that extends the HB toward lower HB masses. We also discuss statistical effects on the metallicity estimate using $P_{0, \min }$ for field RR $a b$ variables reported by Castellani, Maceroni, \& Tosi in 1983. Our calculations suggest that the statistical effect is sufficient to explain the apparent gradient in $P_{0, \min }$ without introducing a metallicity gradient.

Key words: Galaxy: formation - globular clusters: individual (NGC 5272, NGC 7089) -

RR Lyrae variable - stars: horizontal-branch - stars: luminosity function, mass function

\section{INTRODUCTION}

RR Lyrae variables provide crucial information for estimating cluster ages and distances, as nicely summarized by Smith (1995). There are several factors that make RR Lyrae variables potentially good age and distance indicators. They can be easily identified by their distinctive light curves and are bright enough to be observed to considerable distances. They occur in old systems. Finally, their absolute magnitudes appear to be quite restricted. For these reasons, many investigations have exploited these virtues of $R R$ Lyrae variables to estimate ages of globular clusters, for example, with a method that uses the reddeningindependent gap between the RR Lyrae variables or horizontal-branch (HB) stars and the main-sequence turnoff (e.g., Sandage 1982). The key to absolute ages is the value of the absolute magnitude of RR Lyrae variables.

The absolute magnitudes of RR Lyrae variables appear to depend on the metallicity. Considerable work has been directed at determining the dependence, assuming it is linear (e.g., Lee, Demarque, \& Zinn 1990, hereafter LDZ90; Carney, Storm, \& Jones 1992, hereafter CSJ92; Sandage 1993; Fusi Pecci et al. 1996; and Chaboyer et al. 1998). The slope of $M_{V}(\mathrm{RR})-[\mathrm{Fe} / \mathrm{H}]$ relation establishes the relative ages of clusters of differing $[\mathrm{Fe} / \mathrm{H}]$. However, the agreement on the value of the slope of the $M_{V}(\mathrm{RR})-[\mathrm{Fe} / \mathrm{H}]$ may not be the whole story. The potential problem is that the most metal-poor globular clusters are Oosterhoff II clusters, with mean pulsation periods of around 0.65 days for the funda- 
TABLE 1

COMPARISONS BETWEEN M2 AND M3

\begin{tabular}{|c|c|c|c|c|c|}
\hline & & $B-R$ & $n(\mathrm{RR} c)$ & & \\
\hline $\begin{array}{l}\text { Cluster } \\
\text { (1) }\end{array}$ & $\begin{array}{c}{[\mathrm{Fe} / \mathrm{H}]} \\
\text { (2) }\end{array}$ & $\overline{B+\underset{(3)}{V+R}}$ & $\begin{array}{c}n(\mathrm{RR} a b+\mathrm{RR} c) \\
\text { (4) }\end{array}$ & $\begin{array}{c}\left\langle P_{\text {ab }}\right\rangle \\
(5)\end{array}$ & $\begin{array}{c}\langle\beta\rangle \\
(6)\end{array}$ \\
\hline M2 $2 \ldots \ldots$ & -1.62 & 0.92 & 0.40 & 0.685 & 0.06 \\
\hline M3...... & -1.66 & 0.08 & 0.16 & 0.551 & 0.00 \\
\hline
\end{tabular}

REFERENCES.-Col. (2) shows Zinn \& West (1984) metallicity scale; (3), HB color distribution index; (4), number ratio of $\mathrm{RR} c$ type variables to the total number of RR Lyrae type variables; (5), mean period of RRab type variables (day); (6), mean period change rate of RR $a b$ type variables (day $\left.\mathrm{Myr}^{-1}\right)$.

mental mode, compared with the bulk of intermediate metallicity clusters, which are Oosterhoff I clusters, with a mean fundamental-mode pulsation period of around 0.55 days (see the compilation of Castellani \& Quarta 1987). A dichotomy in period distribution may also reflect a dichotomy in RR Lyrae luminosities, as suggested by Clement \& Shelton (1999).

The Oosterhoff dichotomy has been a source of great interest for decades in modern stellar astrophysics and our Galaxy is the only known example showing such a dichotomy. In fact, the RR Lyrae variables in neighboring dwarf galaxies have different values of $\left\langle P_{\mathrm{ab}}\right\rangle$ than either of the Oosterhoff I and II classes. The mean periods of the neighboring dwarfs are: Carina ( 0.62 days; Saha, Monet, \& Seitzer 1986); Draco (0.61 days; Nemec 1985); LMC (0.58 days; Hazen \& Nemec 1992; Alcock et al. 1996); Leo II (0.59 days; van Agt 1973); Sculptor (0.60 days; Goldsmith 1993); and Ursa Minor (0.64 days; Nemec, Wehlau, \& Mendes de Oliveira 1988).

One explanation for the Oosterhoff dichotomy was offered by van Albada \& Baker (1973). They proposed that most of the horizontal-branch stars in intermediate metallicity clusters begin their lives near the instability strip. As they evolve they become hotter, and the fundamental mode RR $a b$ variables would switch to first overtone pulsation $(\mathrm{RR} c)$ when they become hot enough. These would be the Oosterhoff I clusters. The horizontal-branch stars in the more metal-poor clusters begin their lives at higher temperatures, and evolve to lower temperatures. Such stars therefore approach the instability strip from a different direction than those in intermediate metallicity clusters. Further, van Albada \& Baker proposed that there is a hysteresis effect, so that mode switching is delayed and occurs at different temperatures, depending on the direction of evolution. The transition would occur at lower temperatures for stars evolving in that direction than for stars evolving toward higher temperatures. This effect would help explain the larger fraction of RR $c$ variables in Oosterhoff II clusters. The key point is that the "first parameter" would be metallicity, which would determine where on the horizontal-branch core helium burning begins. The more metal-poor clusters have bluer horizontal branches and would become Oosterhoff II clusters. Note that this explanation does not explain the absence of a dichotomy in other galaxies unless the range in metallicity in each galaxy is very small.

Sandage, Katem, \& Sandage (1981) and Sandage (1981, 1982 , 1990b) employed the results of van Albada \& Baker (1973) to demonstrate that the longer periods of the Ooster- hoff II RR $a b$ variables are caused by their higher luminosities. It should be noted, however, that it is the pulsation equation of van Albada \& Baker (1973), not their explanation for the Oosterhoff dichotomy, which leads to this conclusion. While the $M_{V}(\mathrm{RR})$ versus $[\mathrm{Fe} / \mathrm{H}]$ relation of Sandage (1990b) has been questioned because of its consequences in the helium versus metallicity relation (Sweigart, Renzini, \& Tornambé 1987), the inappropriate choice of temperature indicator (CJS92), and the results for M31 globular clusters (Fusi Pecci et al. 1996), the basic idea that the Oosterhoff classes have differing mean luminosities has been confirmed, in that all studies agree that the more metal-poor RR Lyrae variables are brighter.

LDZ90 computed model distributions of horizontalbranch cluster stars for a range of metallicities. Their models spread stars along the horizontal branch by invoking a Gaussian dispersion in mass loss during RGB evolution. Stars with greater mass loss and hence smaller envelope masses begin their core helium burning at higher temperatures, on the blue side of the HB. LDZ90 argued that the Oosterhoff dichotomy likely arises from the "first parameter" effect that leads to bluer horizontal-branch distributions for the lower metallicity Oosterhoff II clusters. Subsequent evolution then favors RR Lyrae variables in Oosterhoff II clusters being more highly evolved and farther from their initial zero-age horizontal-branch (ZAHB) position than the RR Lyrae variables in Oosterhoff I clusters. Qualitatively, the higher luminosities of the Oosterhoff II variables would cause them to have lower densities than Oosterhoff I variables of similar temperatures, and the lower densities would result in longer periods. Quantitatively, the slope of the $M_{V}(\mathrm{RR})$ versus $[\mathrm{Fe} / \mathrm{H}]$ relation predicted by LDZ90 agrees with that obtained by CJS92 and Fusi Pecci et al. (1996).

Clement \& Shelton (1999) have taken an even closer look at the period shift data, using modern CCD-based light curves. They have argued that the amplitude of the $V$ variations, which is a fairly good temperature indicator, does not appear to depend on $[\mathrm{Fe} / \mathrm{H}]$ in general but is related to Oosterhoff classes. They noted that it is therefore possible that two clusters with similar $[\mathrm{Fe} / \mathrm{H}]$ values but belonging to two different Oosterhoff classes may not have the same luminosity and that reliance upon a single $M_{V}(\mathrm{RR})-[\mathrm{Fe} / \mathrm{H}]$ relation may be inappropriate near the metallicity boundary between the two classes.

We pose four key related questions, relevant to the Oosterhoff dichotomy. First, do RR Lyrae variables with similar metallicities in different Oosterhoff classes have the same luminosity? If not, using a single $M_{V}(\mathrm{RR})-[\mathrm{Fe} / \mathrm{H}]$ relation may lead to systematic errors. Second, are the differences related to evolution, as LDZ90 suggested? Third, if evolution is the explanation, what is it that leads to such differences? We argue that a difference in ages is a plausible explanation. Finally, if the Oosterhoff classes differ in age, do they differ in origin as well?

In this paper, we present a comparative study of the Oosterhoff II cluster M2 and the Oosterhoff I cluster M3. Both have similar metallicities, $[\mathrm{Fe} / \mathrm{H}]=-1.62$ for $\mathrm{M} 2$ and -1.66 for M3 (Zinn \& West 1984), but very different HB morphologies: $(B-R) /(B+V+R)=0.92$ for M2 (Lee \& Carney 1999, hereafter LC99) and 0.08 for M3 (Buonanno et al. 1994). These two clusters also exemplify a second parameter pair: clusters with similar $[\mathrm{Fe} / \mathrm{H}]$ values but very different HB morphologies. In $\S 2$, we discuss our database 
for M2 and M3. The metal and helium abundances of two clusters derived using photometric indicators are discussed in $\S 3$. In $\S 4$, we discuss the luminosity differences between M2 and M3 RR Lyrae variables using a period shift analysis, main-sequence (MS) fitting, the luminosity differences between turnoff (TO) and RR Lyrae variables, $\Delta V_{\mathrm{TO}}^{\mathrm{HB}}$ and $\Delta V^{0.05}$, and the luminosity differences between the RGB bump and the RR Lyrae variables, $\Delta V_{\mathrm{ZAHB}}^{\text {bump }}$. In $\S 5$, the effect of an age difference between M2 and M3 on the period differences is explored. We also compare the kinematics of the Oosterhoff group I and II clusters. Finally, we extend the comparison to field stars using an unbiased sample of field RR Lyrae variables with $|Z| \leq 3 \mathrm{kpc}$ and $|Z| \geq 5 \mathrm{kpc}$.

\section{DATA}

In Table 1 , we present $[\mathrm{Fe} / \mathrm{H}]$, the $\mathrm{HB}$ color distribution index, the number ratio of $\mathrm{R} R c$ type variables to the total number of RR Lyrae type variables, the mean period of $\mathrm{RR} a b$ type variables, and the mean period change rate $\langle\beta\rangle$ for M2 and M3. We note that M2 and M3 have similar metallicities, but very different HB morphologies and pulsational properties.

\section{1. $M 2$ (NGC 7089)}

LC99 discusses the observations and the data reductions in detail. The observations were carried out using the $0.9 \mathrm{~m}$ telescopes at Kitt Peak National Observatory (KPNO) and Cerro Tololo Inter-American Observatory (CTIO) ${ }^{1}$ to obtain Johnson $B V$ photometry. The typical exposure times were $360 \mathrm{~s}$ and $240 \mathrm{~s}$ for the long-exposure $B V$ pairs and 20 $\mathrm{s}$ and $15 \mathrm{~s}$ for the short-exposure $B V$ pairs. The field star contamination was removed in a statistical fashion by comparing the color-magnitude diagram (CMD) with off-cluster fields having the same galactic latitude as M2.

The composite M2 CMD is presented in Figure $1 a$. We have used the magnitude-weighted integrated color $(B-V)_{\mathrm{mag}}$ and the intensity-weighted integrated magnitude $\langle V\rangle_{\text {int }}$ for the RR Lyrae variables (see Table 2 of LC99). The $(B-V)_{\text {mag }}$ value gives the closest approximation to the color-temperature relation of the "equivalent static star" (Sandage 1990a). Since the energy generated by nuclear reactions inside a variable star is unaffected by the star's pulsation, $\langle V\rangle_{\text {int }}$ should be the same as would be observed were the star not pulsating (Smith 1995).

We have derived the mean locus for the CMD of M2 using a combination of subjective removal of outliers followed by use of IRAF's CURFIT task. The first step involved removal of stars which, in our consideration, deviate too much from the standard sequences (main sequence, subgiant branch, red giant branch, horizontal branch). Because of the small number of stars, we also drew the red giant branch locus for stars brighter than $V=17$ mag by eye. For the more numerous fainter stars, we followed a more objective procedure.

Since the slopes of color versus magnitude change rapidly near the base of the RGB and near the turnoff, we divided

\footnotetext{
${ }^{1}$ KPNO and CTIO, National Optical Astronomy Observatories, are operated by the Association of Universities for Research in Astronomy, Inc., under cooperative agreement with the National Science Foundation.
}
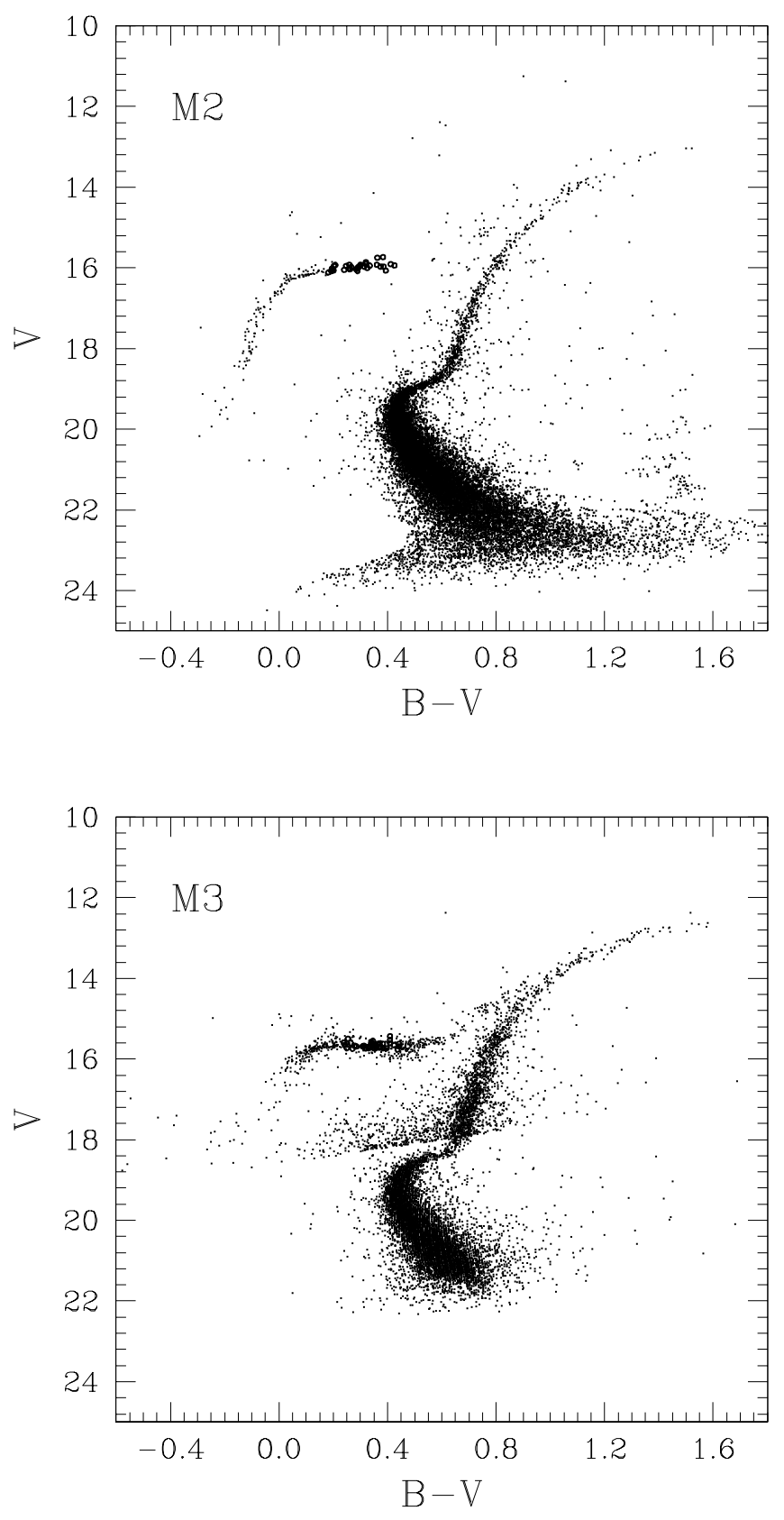

FIG. 1.-Color-magnitude diagrams for the Oosterhoff II cluster M2 (LC99) and the Oosterhoff I cluster M3 (Ferraro et al. 1997; Carretta et al. 1998). The RR Lyrae variables are represented by open circles. The intensity-weighted mean magnitudes are used for RR Lyrae variables. Both clusters have similar metallicities, of $[\mathrm{Fe} / \mathrm{H}]=-1.62$ for $\mathrm{M} 2$ and -1.66 for M3, but very different HB morphologies $(B-R) /$ $(B+V+R)=0.92$ for M2 and 0.08 for M3.

the CMD fitting into three sections: from the RGB tip to the base of the RGB, the base of the RGB to the upper main sequence, and the upper main sequence to the lower main sequence. We included $0.5 \mathrm{mag}$ in overlap for the three fitting regions to ensure continuity. We then performed the curve fitting using modest polynomial orders $\left(\leq 6^{\text {th }}\right)$ and combined the three fitted lines together.

To derive the M2 HB fiducial sequence, we proceeded similarly, using objective methods for high-density regions of the CMD and fitting by eye for the lower density regions. 
We calculated the mean color as a function of $V$ magnitude for a higher CMD star density region [about $16.2<V<18.4$ and $-0.2<(B-V)<0.1]$. We also calculated the $(B-V)_{\mathrm{mag}}$ and $\langle V\rangle_{\mathrm{int}}$ for the M2 RR $c$ and RR $a b$ type variables and used these values for the fiducial sequence of the M2 instability strip. For the rest of the HB region, we read the mean color and $V$ magnitude by eye.

A concern about fitting is the influence of binary stars on the final results. The effects of binaries are more significant for the fainter main-sequence stars, and so we explore this matter in Figure 2, which presents a color distribution around our fiducial sequence near the turnoff point $(19.55<V<19.65)$. The abscissa represents the difference in color between our fiducial sequence and all the observed stars in this magnitude regime. Two points are especially noteworthy. First, it appears that binary systems are present in M2. In the figure, we show the Gaussian fitted curve with $\sigma(B-V)=0.022 \mathrm{mag}$, which has been scaled to have the observed number of stars $(\approx 120$ stars $)$ at $\Delta(B-V)=0.0$. The lower panel shows the difference between the observed distribution and the Gaussian-fitted line. The residual probably represents a binary star population, similar to that found by Bolte (1992) in his study of NGC 288. Thus the presence of binaries could in principle affect the determination of the fiducial sequence in M2 and in M3. Our second point, however, is that the peak of observed color distribution, which presumably reflects that for single stars, is within $0.01 \mathrm{mag}$ in color of our fiducial sequence determined using all the stars. The center of the

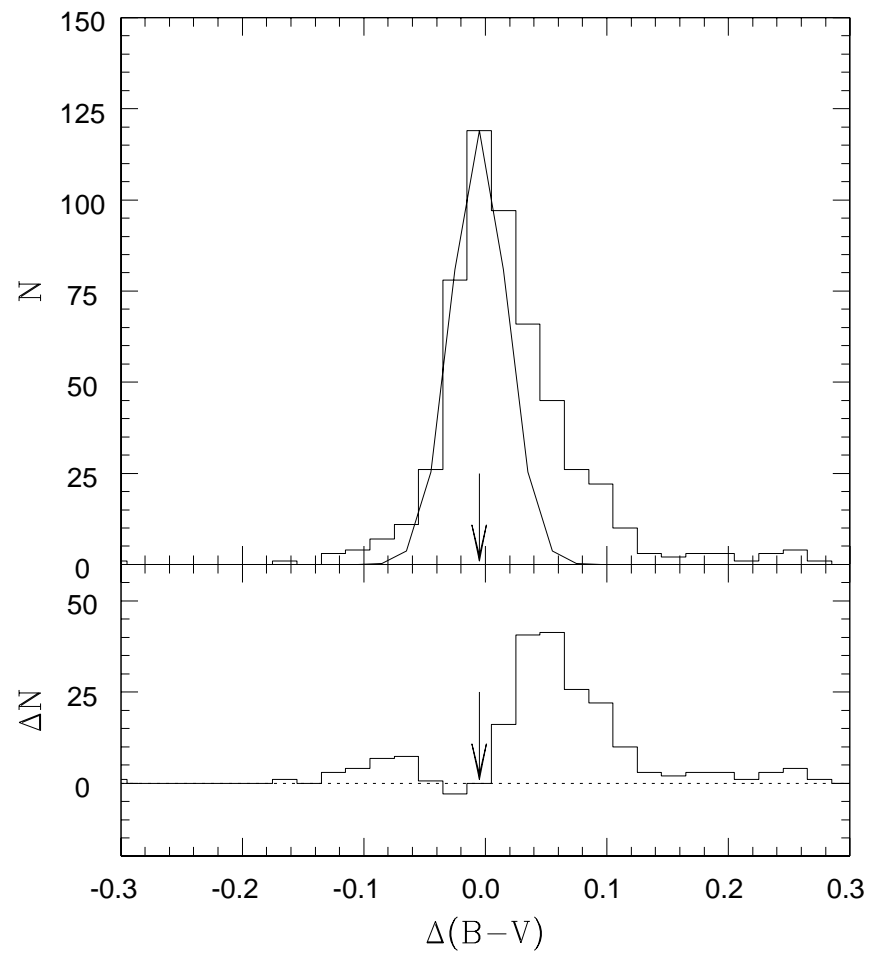

FIG. 2.-Color distribution around the main-sequence fiducial sequence near the turnoff point $(19.55<V<19.65)$. The top panel shows the observed distribution with the Gaussian-fitted line normalized to the number of stars at $\Delta(B-V)=0.0$. The bottom panel shows the difference between the observed distribution and the Gaussian fit. The residual, redder than the main-sequence locus, may represent the contributions of binary systems. The center of the peak of the observed color distribution is indicated by arrows in the figure.
TABLE 2

M2 Fiducial Sequence

\begin{tabular}{|c|c|c|c|}
\hline$V$ & $B-V$ & V & $B-V$ \\
\hline $13.00 \ldots \ldots$ & 1.534 & 18.50 & 0.625 \\
\hline $13.20 \ldots \ldots$ & 1.377 & 18.60 & 0.611 \\
\hline $13.40 \ldots \ldots$ & 1.273 & 18.70 & 0.594 \\
\hline $13.60 \ldots \ldots$ & 1.197 & 18.80 & 0.568 \\
\hline $13.80 \ldots \ldots$ & 1.139 & 18.90 & 0.522 \\
\hline $14.00 \ldots \ldots$ & 1.089 & 19.00 & 0.494 \\
\hline $14.20 \ldots \ldots$ & 1.047 & 19.10 & 0.471 \\
\hline $14.40 \ldots$ & 1.008 & 19.20 & 0.454 \\
\hline $14.60 \ldots \ldots$ & 0.973 & 19.30 & 0.442 \\
\hline $14.80 \ldots$ & 0.941 & 19.40 & 0.437 \\
\hline $15.00 \ldots$ & 0.912 & 19.50 & 0.436 \\
\hline $15.20 \ldots \ldots$ & 0.885 & 19.60 & 0.435 \\
\hline $15.40 \ldots \ldots$ & 0.859 & 19.70 & 0.437 \\
\hline $15.60 \ldots \ldots$ & 0.835 & 19.80 & 0.440 \\
\hline $15.80 \ldots \ldots$ & 0.812 & 19.90 & 0.443 \\
\hline $16.00 \ldots$ & 0.791 & 20.00 & 0.448 \\
\hline $16.20 \ldots \ldots$ & 0.772 & 20.10 & 0.454 \\
\hline $16.40 \ldots \ldots$ & 0.754 & 20.30 & 0.468 \\
\hline $16.60 \ldots \ldots$ & 0.738 & 20.50 & 0.486 \\
\hline $16.80 \ldots \ldots$ & 0.723 & 20.70 & 0.508 \\
\hline $17.00 \ldots \ldots$ & 0.710 & 20.90 & 0.532 \\
\hline $17.20 \ldots \ldots$ & 0.698 & 21.10 & 0.564 \\
\hline $17.40 \ldots \ldots$ & 0.686 & 21.30 & 0.595 \\
\hline $17.60 \ldots \ldots$ & 0.676 & 21.50 & 0.628 \\
\hline $17.80 \ldots \ldots$ & 0.666 & 21.70 & 0.665 \\
\hline $18.00 \ldots \ldots$ & 0.656 & 21.90 & 0.704 \\
\hline $18.20 \ldots \ldots$ & 0.646 & 22.10 & 0.746 \\
\hline $18.30 \ldots \ldots$ & 0.641 & 22.30 & 0.789 \\
\hline $18.40 \ldots \ldots$ & 0.634 & 22.50 & 0.833 \\
\hline
\end{tabular}

peak of the observed color distribution is also indicated by arrows in the figure.

Our smoothed M2 MS and RGB fiducial sequence and unsmoothed HB sequence are given in Tables 2 and 3 and are shown in Figure 3.

Column (9) of Table 2 in LC99 lists the pulsational classes of the M2 RR Lyrae variables. We excluded Blazhko variables (V2, V16, V17, V21, LC184, LC456, and LC798)

TABLE 3

\begin{tabular}{|c|c|}
\hline$V$ & $B-V$ \\
\hline $15.84 \ldots$ & 0.570 \\
\hline $15.94 \ldots$ & 0.364 \\
\hline $16.01 \ldots$ & 0.234 \\
\hline $16.20 \ldots$ & 0.092 \\
\hline $16.40 \ldots$ & 0.024 \\
\hline $16.60 \ldots$ & -0.003 \\
\hline $16.80 \ldots$ & -0.033 \\
\hline $17.00 \ldots \ldots \ldots \ldots$ & -0.050 \\
\hline $17.20 \ldots \ldots \ldots \ldots$ & -0.075 \\
\hline $17.40 \ldots \ldots \ldots \ldots$ & -0.080 \\
\hline $17.60 \ldots \ldots \ldots \ldots$ & -0.089 \\
\hline $17.80 \ldots \ldots \ldots \ldots$ & -0.100 \\
\hline $18.00 \ldots \ldots \ldots \ldots$ & -0.110 \\
\hline $18.20 \ldots \ldots \ldots \ldots$ & -0.125 \\
\hline $18.40 \ldots \ldots \ldots \ldots$ & -0.135 \\
\hline $19.50 \ldots \ldots \ldots \ldots$ & -0.200 \\
\hline $20.20 \ldots \ldots \ldots \ldots$ & -0.272 \\
\hline
\end{tabular}




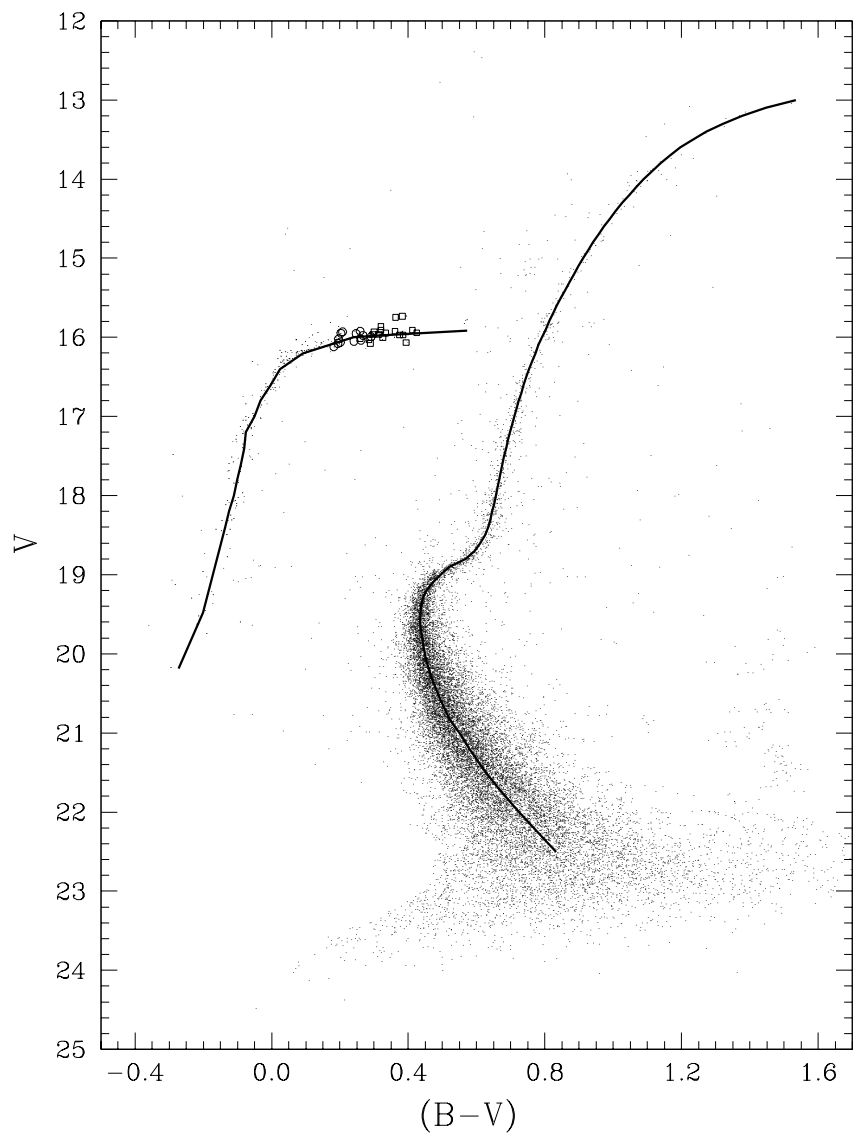

FIG. 3.-Color-magnitude diagram for M2. RRab-type variables are represented by open squares and RR $c$ type variables by open circles. Our M2 fiducial sequences in Table 2 are shown as solid lines.

from our M2 database. The mean intensity-weighted integrated magnitude of the remaining $11 \mathrm{RR} a b$ variables is $\left\langle V_{\mathrm{RR}}\right\rangle=15.926 \pm 0.021 \mathrm{mag}$ in the $V$ bandpass. (The error is that of the mean.)

\section{2. $M 3(N G C 5272)$}

Ferraro et al. (1997) obtained BVI CCD photometry of M3 using the $3.6 \mathrm{~m}$ Canada-France-Hawaii telescope and they presented the CMD in their Figure 5, combined with the recalibrated older photographic study of Buonanno et al. (1994). We reproduce their CMD in Figure $1 b$ of this paper. Although they have presented nice fiducial sequences of M3 in their Table 3, we reanalyzed their data using our procedure in order to prevent any possible systematic effects. We obtained their photometric results via the SIMBAD $^{2}$ database, and we rederived the fiducial sequence for M3 main-sequence and RGB stars using the methods described above. Our smoothed fiducial sequence for M3 is given in Table 4.

Carretta et al. (1998) presented new BVI CCD photometry of 60 RR Lyrae variables in M3. As they noted, their photometric calibration is tied directly to that of Ferraro et al. (1997). In order to construct our database for M3 RRab variables, we examined the light curves given by Carretta et al. (1998). We excluded Blazhko variables and variables with an incomplete light curve near the maximum or minimum (V27, V34, V43, V74, V121, V143, and V197) from our M3 database. The mean intensity-weighted integrated

\footnotetext{
${ }^{2}$ The SIMBAD database is operated at CDS, Strasbourg, France.
}

TABLE 4

M3 Fiducial Sequence

\begin{tabular}{|c|c|c|c|}
\hline$V$ & $B-V$ & V & $B-V$ \\
\hline $12.60 \ldots \ldots$ & 1.579 & 18.10 & 0.654 \\
\hline $12.80 \ldots \ldots$ & 1.440 & 18.20 & 0.633 \\
\hline $13.00 \ldots \ldots$ & 1.310 & 18.30 & 0.603 \\
\hline $13.20 \ldots \ldots$ & 1.229 & 18.40 & 0.568 \\
\hline $13.40 \ldots \ldots$ & 1.159 & 18.50 & 0.533 \\
\hline $13.60 \ldots \ldots$ & 1.098 & 18.60 & 0.502 \\
\hline $13.80 \ldots \ldots$ & 1.046 & 18.70 & 0.478 \\
\hline $14.00 \ldots \ldots$ & 1.000 & 18.80 & 0.461 \\
\hline $14.20 \ldots \ldots$ & 0.961 & 18.90 & 0.451 \\
\hline $14.40 \ldots \ldots$ & 0.927 & 19.00 & 0.446 \\
\hline $14.60 .$. & 0.898 & 19.10 & 0.444 \\
\hline $14.80 \ldots \ldots$ & 0.872 & 19.20 & 0.445 \\
\hline $15.00 \ldots \ldots$ & 0.849 & 19.30 & 0.446 \\
\hline $15.20 \ldots \ldots$ & 0.829 & 19.40 & 0.448 \\
\hline $15.40 \ldots \ldots$ & 0.812 & 19.50 & 0.453 \\
\hline $15.60 \ldots \ldots$ & 0.796 & 19.60 & 0.459 \\
\hline $15.80 \ldots \ldots$ & 0.782 & 19.70 & 0.465 \\
\hline $16.00 \ldots \ldots$ & 0.769 & 19.80 & 0.471 \\
\hline $16.20 \ldots \ldots$ & 0.757 & 19.90 & 0.480 \\
\hline $16.40 \ldots \ldots$ & 0.745 & 20.00 & 0.490 \\
\hline $16.60 \ldots \ldots$ & 0.735 & 20.20 & 0.512 \\
\hline $16.80 \ldots \ldots$ & 0.724 & 20.40 & 0.535 \\
\hline $17.00 \ldots$ & 0.714 & 20.60 & 0.560 \\
\hline $17.20 \ldots \ldots$ & 0.705 & 20.80 & 0.586 \\
\hline $17.40 \ldots \ldots$ & 0.695 & 21.00 & 0.615 \\
\hline $17.60 \ldots \ldots$ & 0.685 & 21.20 & 0.645 \\
\hline $17.80 \ldots \ldots$ & 0.672 & 21.40 & 0.678 \\
\hline $17.90 \ldots \ldots$ & 0.670 & 21.70 & 0.737 \\
\hline $18.00 \ldots \ldots$ & 0.666 & 21.90 & 0.773 \\
\hline
\end{tabular}

magnitude of $35 \mathrm{RR} a b$ variables is $\left\langle V_{\mathrm{RR}}\right\rangle=15.665 \pm 0.013$ mag in the $V$ bandpass. (The error is that of the mean.)

\section{METALLICITY AND HELIUM ABUNDANCE}

We estimate the metallicities of M2 and M3 based on two photometric indicators: $(B-V)_{0, g}$ (Sandage \& Smith 1966) and $\Delta V_{1.4}$ (Sandage \& Wallerstein 1960), using the fiducial sequences in Tables 2, 3, and 4 . We use the mean $V$ magnitude of RR Lyrae variables $\left\langle V_{\mathrm{RR}}\right\rangle$ discussed above as the luminosity level of HB.

The index $(B-V)_{0, q}$ is the dereddened $(B-V)$ color of the RGB at the luminosity level of HB stars in the CMD. We find $\left\langle V_{\mathrm{RR}}\right\rangle_{0}=15.864 \mathrm{mag}$ for M2 and $15.634 \mathrm{mag}$ for M3, assuming the interstellar absorption in $V$ bandpass is $A_{V}=3.1 E(B-V)$ and $E(B-V)=0.02$ for $\mathrm{M} 2$ and $E(B-V)=0.01$ for M3 (Zinn 1985), where $\left\langle V_{\mathrm{RR}}\right\rangle_{0}$ refers to the interstellar absorption corrected mean $V$ magnitude of RR Lyrae variables. By interpolating the fiducial sequences of M2 and M3, we obtain $(B-V)_{0, g}=0.78 \pm 0.03 \mathrm{mag}$ for $\mathrm{M} 2$ and $0.78 \pm 0.02 \mathrm{mag}$ for $\mathrm{M} 3$. Using the relation given by Ferraro et al. (1997)

$$
[\mathrm{Fe} / \mathrm{H}]_{\mathrm{Zw} 84}=4.30(B-V)_{0, g}-5.00,
$$

we obtain $[\mathrm{Fe} / \mathrm{H}]=-1.65$ for $\mathrm{M} 2$ and -1.65 for M3 using the Zinn \& West (1984) metallicity scale.

Carretta \& Bragaglia (1998) provide a new calibration of the $(B-V)_{0, g}$ indicator based on the revised metallicity scale obtained by Carretta \& Gratton (1997). Using their new relation

$$
\begin{aligned}
{[\mathrm{Fe} / \mathrm{H}]_{\mathrm{CG} 97}=} & 20.129(B-V)_{0, g}-9.253(B-V)_{0, g}^{2} \\
& -11.532,
\end{aligned}
$$


we find $[\mathrm{Fe} / \mathrm{H}]=-1.46$ for $\mathrm{M} 2$ and -1.46 for $\mathrm{M} 3$ in the Carretta \& Gratton (1997) metallicity scale.

The index $\Delta V_{1.4}$ is defined to be the difference in $V$ magnitude between the $\mathrm{HB}$ and the RGB at $(B-V)_{0}=1.40$. By interpolating the dereddened fiducial sequences, we obtain $V_{1.4}=13.08 \mathrm{mag}$ for M2 and $12.82 \mathrm{mag}$ for M3, where $V_{1.4}$ refers to the interstellar absorption corrected $V$ magnitude of RGB at $(B-V)_{0}=1.40 \mathrm{mag}$. Therefore, $\Delta V_{1.4}$ becomes $2.78 \mathrm{mag}$ for M2 and $2.81 \mathrm{mag}$ for M3. Using the relation given by Ferraro et al. (1997)

$$
[\mathrm{Fe} / \mathrm{H}]_{\mathrm{zw} 84}=-0.924 \Delta V_{1.4}+0.913,
$$

we obtain $[\mathrm{Fe} / \mathrm{H}]=-1.66$ for $\mathrm{M} 2$ and -1.68 for $\mathrm{M} 3$ in the Zinn \& West (1984) scale. Our metal abundance estimations using photometric indicators show that M2 and M3 have very similar metallicities, confirming the previous results of Zinn \& West (1984).

In a forthcoming paper, we will present an abundance analysis of M2. We obtained high-resolution $(R=28,000)$ and high signal-to-noise ratio $(>100)$ echelle spectra for two M2 red giants using the CTIO $4 \mathrm{~m}$ telescope and its Cassegrain echelle spectrograph. Our preliminary result indicates that the $[\mathrm{Fe} / \mathrm{H}]$ of the $\mathrm{M} 2$ red giants agree well with that of M3 (Kraft et al. 1992)-within 0.1 dex in the sense that M2 is slightly more metal-poor, which is in agreement with the photometric results discussed above. We find the abundances of the " $\alpha$ " elements (silicon, magnesium, calcium, and titanium) relative to iron are also similar in the two clusters.

We compare the helium abundance of M2 and M3 using the R method (Buzzoni et al. 1983; Caputo, Roger, \& Paez 1987). We use $V_{\mathrm{ZAHB}}$ as the luminosity of the HB stars as recommended by Buzzoni et al. (1983). We derived the $V_{\mathrm{ZAHB}}$ value using the relation given by CSJ92) to correct for the vertical height of the HB stars

$$
V_{\mathrm{ZAHB}}=\left\langle V_{\mathrm{RR}}\right\rangle+0.05[\mathrm{Fe} / \mathrm{H}]+0.20,
$$

where $\left\langle V_{R R}\right\rangle$ is the mean visual magnitude of the RR Lyrae variables. From the mean magnitude of $11 \mathrm{RR} a b$ variables in M2, we obtain $V_{\mathrm{ZAHB}, \mathrm{M} 2}=16.05 \pm 0.02 \mathrm{mag}$. By counting stars with $r>90^{\prime \prime}$ only to avoid crowding-induced incompleteness, we obtain $N_{\mathrm{HB}}=262$ (see LC99), $N_{\mathrm{RGB}}=$ 192 , and $N_{\mathrm{AGB}}=46$ for $\mathrm{M} 2$, where $N_{\mathrm{RGB}}$ refers to the number of RGB stars brighter than $V_{\mathrm{ZAHB}}$. Thus the number ratio of RGB stars and $\mathrm{HB}$ stars is $R=N_{\mathrm{HB}} / N_{\mathrm{RGB}}=1.36$, and the number ratio of $\mathrm{RGB} / \mathrm{AGB}$ stars and $\mathrm{HB}$ stars is $R^{\prime}=N_{\mathrm{HB}} /\left[N_{\mathrm{RGB}}+N_{\mathrm{AGB}}\right]=1.10$. We obtain the helium abundances based on $R$ and $R^{\prime}, Y_{R}=0.23$, and $Y_{R^{\prime}}=0.22$, using equations (11) and (12) of Buzzoni et al. (1983). Our helium abundance estimation is in excellent agreement with their mean value $Y=0.23 \pm 0.02$.

For the M3 helium abundance, we simply adopted the result given by Ferraro et al. (1997). They obtained $R=1.32$ and $R^{\prime}=1.14$, and the estimated helium abundances for M3 are $Y_{R}=0.22$ and $Y_{R^{\prime}}=0.23$, respectively.

In summary, M2 and M3 have very similar metallicities and helium abundances.

\section{RR LYRAE LUMINOSITY COMPARISONS}

\subsection{Period Shift Analysis}

The period shift is the difference between the periods of RR Lyrae variables at fixed temperatures, the comparisons usually being made between globular clusters' variables (Sandage 1981; Sandage et al. 1981; CSJ92; Sandage 1993).
The period-density relation states that the period of a pulsating star is inversely proportional to the square root of the mean density. Assuming the same mass and the same effective temperature, the less dense star would have a longer period. Qualitatively, at a fixed temperature and mass, a lower density star has a larger radius and hence a greater luminosity. More precisely, we adopt the relations given by van Albada \& Baker (1971). For the fundamental mode, the period of an RR Lyrae variable is

$$
\begin{aligned}
\log P_{0}= & -1.772-0.68 \log \frac{M}{M_{\odot}}+0.84 \log \frac{L}{L_{\odot}} \\
& +3.48 \log \frac{6500}{T_{\text {eff }}} .
\end{aligned}
$$

Unfortunately, however, the effective temperature of a star is not an observable parameter. One needs, therefore, other observable parameters that can be related to the effective temperature. As noted in their pioneering paper, Sandage et al. (1981) showed that the blue amplitude $A_{B}$ may be a good indicator of the effective temperature of RR Lyrae variables. The advantage of using parameters such as $P_{0}$ and $A_{B}$ is that these can be measured with a great accuracy and independently of both the distance and the interstellar reddening.

CSJ92 applied the results of Baade-Wesselink analyses and derived relations between pulsational parameters and the equilibrium temperature for the R R $a b$ variables in their equations (13)-(16). Catelan (1998) derived a revised relation excluding $\log P_{0}$ in his period shift analyses of field and cluster variables, finding

$$
\Theta_{\text {eq }}=0.868-0.084 A_{B}+0.005[\mathrm{Fe} / \mathrm{H}],
$$

where $\Theta_{\text {eq }}=5040 / T_{\text {eq }}$, and $T_{\text {eq }}$ is the equilibrium temperature. The equilibrium temperature is not the same as

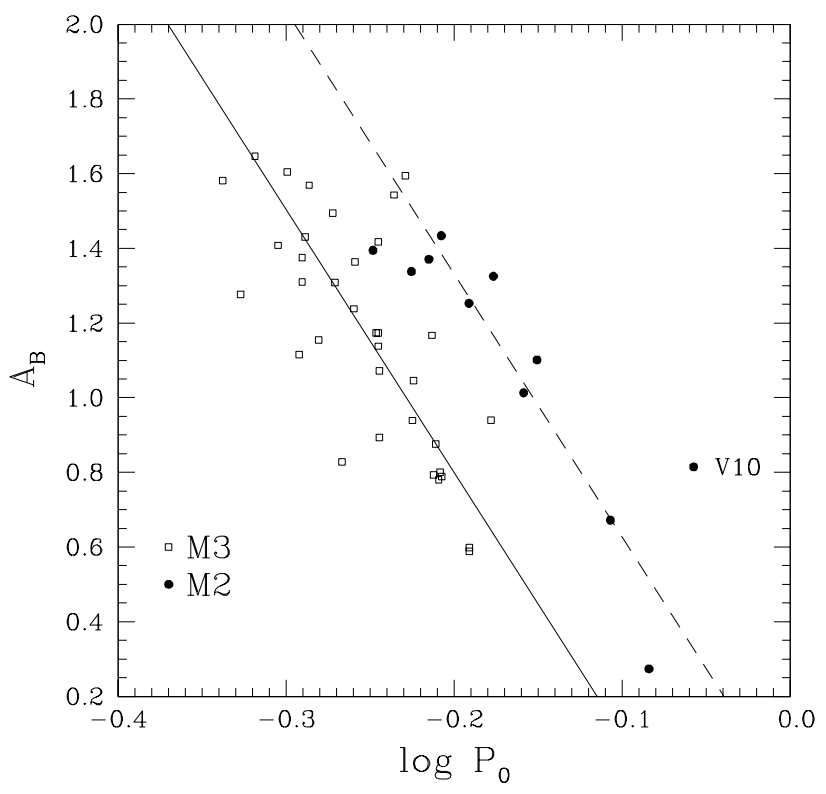

FIG. 4.-Comparison of $\log P_{0}$ vs. $A_{B}$ for M2 and M3 RRab variables. The M2 RR Lyrae variables are represented by filled circles and M3 RR Lyrae variables by open squares. The solid line is the fitted line of M3 RR Lyrae variables and the dashed line is the fitted line of M2 RR Lyrae variables using the same slope as that for M3. V10 in M2 has an unusually long period compared with other variables in M2. The period shift analysis within M2 variables predicts V10 should be 0.24 mag brighter than the other variables in M2. 
TABLE 5

DIFFERENCES OF RR LYRAE RELATED LUMINOSITIES BETWEEN M2 AND M3

\begin{tabular}{ccc}
\hline \hline Method & $\Delta M_{V}(\mathrm{M} 2-\mathrm{M} 3)$ & $\sigma(V)$ \\
\hline Period shift $\ldots \ldots \ldots \ldots \ldots$ & 0.23 & 0.03 \\
MS fitting $\ldots \ldots \ldots \ldots \ldots$ & 0.18 & 0.03 \\
$\Delta V_{\mathrm{TO}}^{\mathrm{HB}} \ldots \ldots \ldots \ldots \ldots \ldots \ldots$ & 0.23 & 0.14 \\
$\Delta V^{0.05} \ldots \ldots \ldots \ldots \ldots \ldots$ & 0.19 & 0.14 \\
$\Delta V_{\mathrm{ZAHB}}^{\text {bump }} \ldots \ldots \ldots \ldots \ldots \ldots$ & 0.13 & 0.07 \\
\hline
\end{tabular}

the effective temperature $T_{\text {eff }}$, but CSJ92 showed that they are very similar. It should be noted that the relation above is rather insensitive to the metallicity, and the effect is minimized further here because M2 and M3 have almost identical metallicities.

Figure 4 presents a $\log P_{0}$ versus $A_{B}$ diagram for M2 and M3. The linear fitted lines are also shown. In order to derive the linear fitted lines, we employed the bissector linear fitting method given by Isobe et al. (1990), which takes the projection vectors normal to the linear fitted line and finds the fitted lines by minimizing the residuals in both axes. We found the linear relation for the M3 RR $a b$ variables first, and then we found the best line for those in M2 with the same slope found in M3 by minimizing residuals in both $\log P_{0}$ and $A_{B}$. We calculated the period shift between M2 and M3 RR Lyrae variables: $\Delta \log P_{0}$ $(\mathrm{M} 3-\mathrm{M} 2)=-0.0771 \pm 0.0114$. We apply this period shift in equation (5) and then calculate $\Delta \log L$ between M2 and M3. Assuming the same surface gravities, we obtain $\Delta M_{V}$ from their luminosity differences, since they have similar temperatures and metallicities hence the bolometric corrections should be the same. We list the $\Delta M_{V}$ value between M2 and M3 RR Lyrae variables based on the period shift analysis in Table 5. The $\Delta M_{V}$ of individual RR Lyrae variables in M2 and M3 are presented in Figure $5 a$ as well. The RR Lyrae magnitude difference between M2 and $\mathrm{M} 3$ is $\Delta M_{V}(\mathrm{RR})=0.229 \pm 0.034 \mathrm{mag}$, in the sense that M2 RR Lyrae variables are more luminous than those in M3. It should be noted that the variable V10 in M2 has an unusually long period compared with other variables in the cluster in Figure 4, and a period shift analysis within M2 above predicts V10 should be $0.24 \pm 0.06$ mag brighter than the other variables in Figure 5a. The results of LC99 show that V10 is, in fact, $0.21 \pm 0.03 \mathrm{mag}$ brighter than those variables (see Figure $5 b$ ). The period shift method appears to be reliable.

We also compared the period shift of M2 with one other Oosterhoff II cluster, NGC 5986 (Liller \& Lichten 1978; $[\mathrm{Fe} / \mathrm{H}]=-1.67$, Zinn \& West 1984), and with two more Oosterhoff I clusters having metallicities similar to M2, NGC 3201 (Cacciari 1984; $[\mathrm{Fe} / \mathrm{H}]=-1.61,{ }^{3}$ Zinn \& West 1984) and NGC 7006 (Wehlau et al. 1992, 1999; [Fe/ $\mathrm{H}]=-1.59^{3}$; Zinn \& West 1984). We obtained $\Delta M_{V}(\mathrm{RR})=0.017 \pm 0.078 \mathrm{mag}$ in comparison with the Oosterhoff II cluster NGC 5986, in the sense that M2 RR Lyrae variables are negligibly brighter. For the Oosterhoff I clusters, we obtain $\Delta M_{V}(\mathrm{RR})=0.229 \pm 0.033 \mathrm{mag}$ for NGC 3201 and $0.280 \pm 0.031 \mathrm{mag}$ for NGC 7006, in the

\footnotetext{
${ }^{3}$ The high-resolution spectroscopic abundance analysis of Kraft et al. (1998) showed that metallicity of NGC 7006 is $[\mathrm{Fe} / \mathrm{H}]=-1.55$. Their measurement of the metal abundance for $\mathrm{M} 3$ is $[\mathrm{Fe} / \mathrm{H}]=-1.47$. Gonzalez \& Wallerstein (1998) found $[\mathrm{Fe} / \mathrm{H}]=-1.42$ for NGC 3201.
}
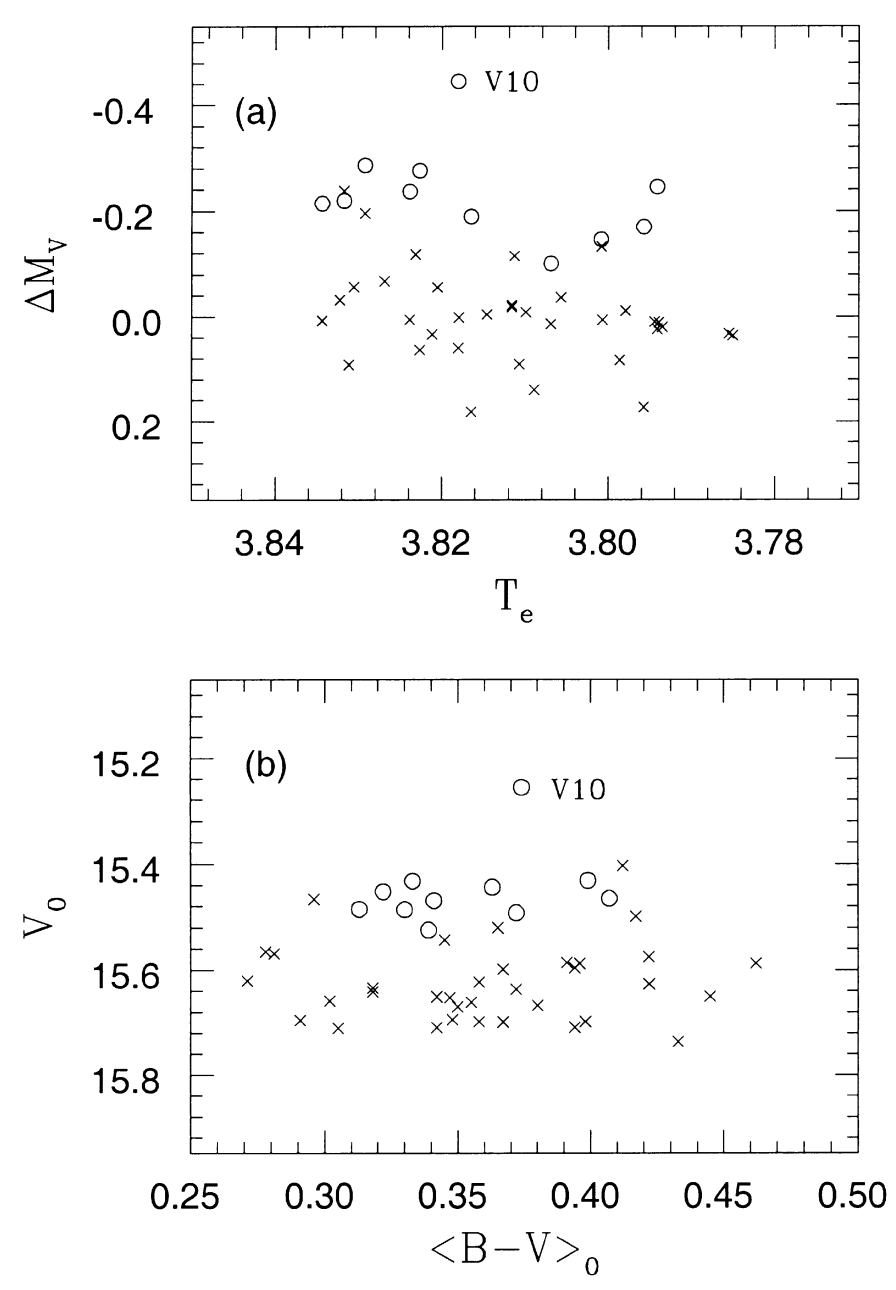

FIG. 5.-Luminosity differences between M2 and M3 RR $a b$ variables determined by $(a)$ period shift analysis and $(b)$ main-sequence fitting. M2 RR Lyrae variables are represented by open circles and M3 RR Lyrae variables by crosses. The zero point is set to the mean magnitude of M3 RR variables in $(a)$. The relative distance modulus of $\Delta(m-M)_{\mathrm{M} 2-\mathrm{M} 3}=$ $0.415 \pm 0.021$ is adopted in $(b)$. In $(b)$, V10 is 0.21 mag brighter than the other variables in M2.

sense that M2 RR Lyrae variables are significantly brighter than those in the Oosterhoff I clusters as we discussed in comparison with the M3 RR Lyrae variables. Our period shift analyses therefore indicate that the Oosterhoff II clusters, M2 and NGC 5986, have similar RR Lyrae luminosities and both clusters' variables are more luminous than those of Oosterhoff I clusters having similar metallicities.

\subsection{Main-Sequence Fitting}

The luminosity difference of the RR Lyrae variables obtained via the period shift analysis is inferential and depends on some uncertain physical parameters, such as stellar mass. A more direct comparison may be achieved by comparing the results from the main-sequence fitting. Therefore we carry out such an analysis fitting using the fiducial sequences that we obtained in $\S 2$.

We compare the M2 and M3 fiducial sequences using less evolved main-sequence stars, those with $(B-V)_{0}$ colors about from 0.6 to $0.7 \mathrm{mag}$. By comparing the M2 and M3 fiducial sequences, we obtain the relative distance modulus between two clusters, $\Delta(m-M)_{\mathrm{M} 2-\mathrm{M} 3}=0.415 \pm 0.021 \mathrm{mag}$ (the error does not include the uncertainties of each fiducial 
sequence). Applying this relative distance modulus to the visual magnitudes of the RR Lyrae variables in M2, we calculate the RR Lyrae absolute magnitude differences between M2 and M3. For this calculation, we use only the $\mathrm{RR} a b$ variables used in the period shift analysis in order to compare the difference between two methods, and we adopt the intensity-weighted integrated magnitudes (col. [7] in Table 2 of LC99 and col. [11] in Table 3 of Carretta et al. 1998). Our result is $\Delta M_{V}(\mathrm{RR} a b)=0.175 \pm 0.025 \mathrm{mag}$ (the error is that of the mean), in the sense that M2 RR Lyrae variables are more luminous than those of M3, consistent with the period shift analysis results. The luminosity differences of the individual RR Lyrae variables based on the main-sequence fitting are presented in Figure $5 b$.

\section{3. $\Delta V_{\mathrm{TO}}^{\mathrm{HB}}$ and $\Delta V^{0.05}$}

The index $\Delta V_{\mathrm{TO}}^{\mathrm{HB}}$ is the difference in luminosity between the HB and the main-sequence turnoff (Sandage 1982) and is an important indicator of age for globular clusters. In recent years, the $\Delta V_{\mathrm{TO}}^{\mathrm{HB}}$ method has become one of the most widely used techniques for dating clusters (Sarajedini, Chaboyer, \& Demarque 1997). It is independent of the cluster reddening and distance, but is, as we noted, dependent on the slope of the adopted relation between $M_{V}(\mathrm{RR})$ and $[\mathrm{Fe} / \mathrm{H}]$.

We measured the turnoff magnitudes, which are $V_{\mathrm{TO}}=$ $19.60 \pm 0.10 \mathrm{mag}$ for M2 and $19.10 \pm 0.10 \mathrm{mag}$ for M3. Using the $\left\langle V_{\mathrm{RR}}\right\rangle$ values from $\S 2$, we obtain $\Delta V_{\mathrm{TO}}^{\mathrm{HB}}(\mathrm{M} 2)=$ $3.67 \pm 0.10 \mathrm{mag}$ and $\Delta V_{\mathrm{TO}}^{\mathrm{HB}}(\mathrm{M} 3)=3.44 \pm 0.10 \mathrm{mag}$. The gap between the horizontal branch and the main-sequence turnoff is about $0.2 \mathrm{mag}$ larger in M2 than in M3.

In practice, the $\Delta V_{\mathrm{TO}}^{\mathrm{HB}}$ method technique has difficulty precisely locating the turnoff luminosity, because the CMD sequence is nearly vertical near the turnoff (Sarajedini et al. 1997; Buonanno et al. 1998). The index $\Delta V^{0.05}$ is the difference in magnitude between the upper main-sequence at a point $0.05 \mathrm{mag}$ redder than the main-sequence turnoff $\left(V_{+0.05}\right)$ and the HB (Buonanno et al. 1998). Near the $V_{+0.05}$ point, the CMD sequence is less steep, and it is easier to determine its magnitude. It shares with the $\Delta V_{\mathrm{TO}}^{\mathrm{HB}}$ method the same theoretical background and is also independent of distance and reddening.

By interpolating the fiducial sequences, we obtain $V_{+0.05}=20.50 \mathrm{mag}$ for $\mathrm{M} 2$ and $V_{+0.05}=20.05 \mathrm{mag}$ for M3. We thereby obtain $\Delta V^{0.05}(\mathrm{M} 2)=4.57 \pm 0.10 \mathrm{mag}$ and $\Delta V^{0.05}(\mathrm{M} 3)=4.38 \pm 0.10$ mag. We find again that the gap between the horizontal branch and the main-sequence turnoff region is about 0.2 mag larger in M2 than in M3. However, we must be careful in comparing these differences to the results from our period shift analysis and from mainsequence fitting using cooler, unevolved stars. $\Delta V_{\mathrm{TO}}^{\mathrm{HB}}$ and $\Delta V^{0.05}$ are affected by differences in cluster ages as well as differences in HB luminosities, while the period shift analysis and main-sequence fitting are not strongly affected by age differences.

\section{4. $\Delta V_{\mathrm{ZAHB}}^{\mathrm{bump}}$}

In some globular clusters, the luminosity function (LF) of the RGB shows a clump of stars, the so-called RGB bump. This clump originates from the drop in luminosity, and hence slower evolution, experienced by RGB stars when the very thin H-burning shell crosses the discontinuity in the chemical composition and lowered mean molecular weight left by the deepest penetration of the convective envelope
(Renzini \& Fusi Pecci 1988; Fusi Pecci et al. 1990; Bono \& Castellani 1992; Chiosi, Bertelli, \& Bressan 1992; Cassisi, Degl'Innocenti, \& Salaris 1997; Cassisi \& Salaris 1997; Alves \& Sarajedini 1999). Since this bump exhibits a break of slope in the cumulative LF, the cumulative LF is known to be a useful tool to detect the RGB bump.

Ferraro et al. (1997) detected the RGB bump in M3 (see their Figs. $17 a$ and $17 b$ ) at $V_{\text {bump }}=15.45 \pm 0.05 \mathrm{mag}$. In Figure 6, we present the differential and the cumulative LFs for the M2 RGB and main-sequence stars with a binning size of 0.05 mag. As can be seen, there is an RGB bump at $V_{\text {bump }}=15.85 \pm 0.05 \mathrm{mag}$ (the quoted error is the binning size of the LF). At $V=15.85 \mathrm{mag}$, the RGB is well separated in color from the $\mathrm{HB}$ or the AGB, hence there is no confusion in counting only RGB stars. The field star contamination is almost negligible, since M2 lies at a fairly higher galactic latitude $\left(b=-36^{\circ}\right)$ and the field stars were removed beforehand in a statistical fashion. The estimated number of field stars toward M2 by Ratnatunga \& Bahcall (1985) is $8.8 \times 10^{-2}$ stars $\operatorname{arcmin}^{-2}$ within the range of $15 \leq$ (apparent visual magnitude) $\leq 17$ and $0.8 \leq(B-V) \leq 1.3$ and is negligibly small, in any case. However, there is a another factor that may affect the LF: blending. We have selected stars with $r>90^{\prime \prime}$ only to avoid the crowding-induced incompleteness. Further, blending is more a problem for the fainter stars and not for the stars as bright as $\mathrm{V} \approx 16 \mathrm{mag}$. Therefore the presence of the RGB bump in $\mathrm{M} 2$ is probably real.

The index $\Delta V_{\mathrm{ZAHB}}^{\mathrm{bump}}$ is defined to be the magnitude difference between the RGB bump and the ZAHB, $\Delta V_{\mathrm{ZAHB}}^{\text {bump }}=$ $V_{\text {bump }}-V_{\mathrm{ZAHB}}$. Using the $V_{\mathrm{ZAHB}}$ values discussed in $\S 3$, we obtain $\Delta V_{\mathrm{ZAHB}}^{\text {bump }}=-0.20 \pm 0.05 \mathrm{mag}$ for $\mathrm{M} 2$ and

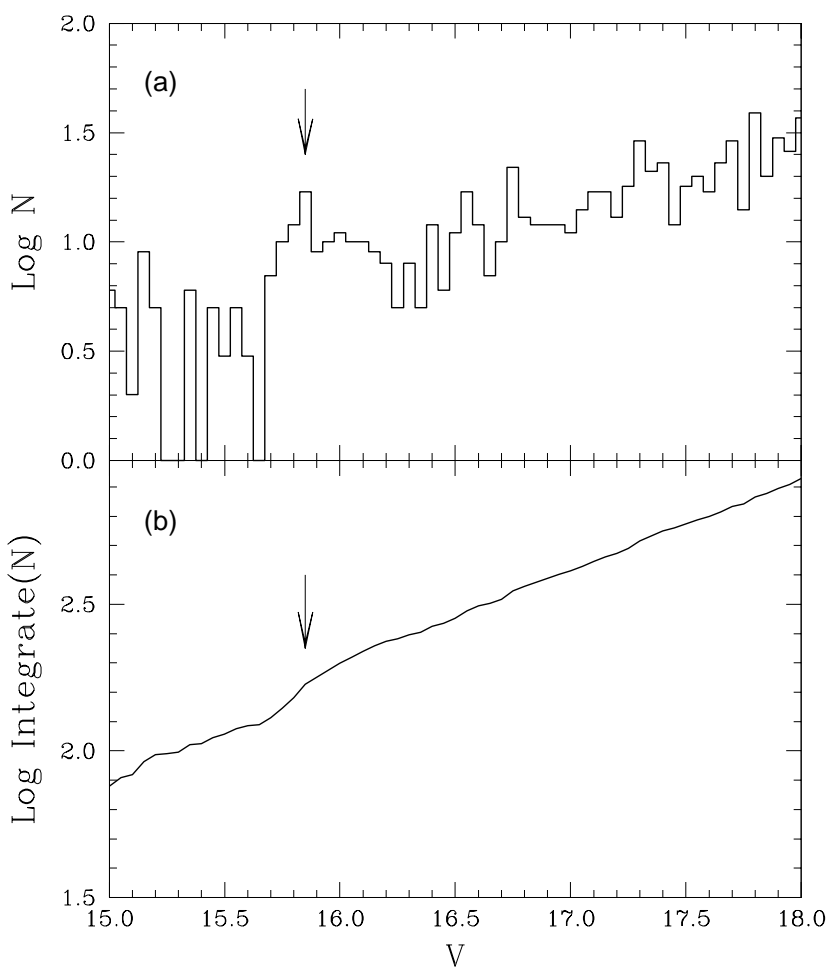

FIG. 6.-Differential luminosity function $(a)$ and cumulative luminosity function $(b)$ of M2. The location of the RGB bump at $V_{\text {bump }}=15.85$ is indicated by arrows. The differential luminosity function shows a peak at the RGB bump and the cumulative luminosity function shows a break of slope at the RGB bump. 
TABLE 6

COMPARISONS OF M2 AND M3

\begin{tabular}{lccc}
\hline \hline Parameter & M2 & M3 & $\sigma(V)$ \\
\hline$V_{\text {bump }} \cdots \cdots$ & 15.85 & 15.45 & 0.05 \\
$V_{\text {RR }} \cdots \cdots \cdots$ & 15.93 & 15.67 & 0.02 \\
$V_{\text {TO }} \ldots \ldots \ldots$ & 19.60 & 19.10 & 0.10 \\
$V_{+0.05} \cdots \cdots$ & 20.50 & 20.05 & 0.10 \\
\hline
\end{tabular}

$\Delta V_{\mathrm{ZAHB}}^{\text {bump }}=-0.33 \pm 0.05$ mag for M3. The gap between the horizontal branch and the RGB bump is about $0.13 \mathrm{mag}$ larger in M2 than in M3, despite their similar metallicities.

In Table 5, we summarize the RR Lyrae related luminosity differences between M2 and M3. In Table 6, we present the list of magnitudes we have discussed in this section. We conclude that the $R R$ Lyrae variables in the Oosterhoff group II cluster M2 are intrinsically brighter than those in Oosterhoff group I cluster M3. Thus at least in this cluster pair (and apparently also for M2 and NGC 5086 in comparison with M3, NGC 3201 and NGC 7006) there is a discontinuity in the $M_{V}(\mathrm{RR})-[\mathrm{Fe} / \mathrm{H}]$ relation.

We explore next what might cause such differences despite similarities in chemical composition.

\section{DISCUSSION}

\subsection{Could the Luminosity Differences Be Caused By Differences in Evolutionary State?}

The HB models of Lee \& Demarque (1990), Dorman (1992), and Yi, Demarque, \& Kim (1997) predict that the metallicity, helium abundance, and mass of HB stars may affect the HB morphology and luminosity. M2 and M3 have similar metallicities and helium abundances, and so cannot easily explain the differences in HB morphologies or luminosities. The mean mass of the HB stars is related to the mass at the RGB tip, which depends on the age of the clusters for a given chemical composition. It also depends on the mass loss at the RGB tip, since enhanced mass loss can produce a bluer HB (Sweigart 1997).

The luminosity of HB stars also depends on their evolutionary state. As an HB star evolves away from its ZAHB, it becomes more luminous. So our derived differences in the luminosity of RR Lyrae variables might imply that M2 RR Lyrae variables are more evolved. The bluer HB morphology $[(B-R) /(B+V+R)=0.92]$ and the higher mean period change rate of the M2 RR Lyrae variables, $\langle\beta\rangle=0.06 \pm 0.04\left(d \mathrm{Myr}^{-1}\right)$ (LC99), indicate that the M2 RR Lyrae variables have evolved significantly away from their ZAHB locations, which are presumably hotter than the instability strip, and to lower densities. The M3 RR Lyrae variables show a smaller period change rate, $\langle\beta\rangle=0.00 \pm 0.03\left(d \mathrm{Myr}^{-1}\right)$ (see, e.g., Figure 10 of LC99 and references therein). This is consistent with the M3 RR Lyrae variables being in a slower stage of evolution and hence closer to their ZAHB locations (Lee 1991). Therefore the existence of the RR Lyrae luminosity differences between M2 and M3 may indeed be caused by differences in the HB evolutionary states of the stars.

\subsection{Could the Evolutionary Differences Be Due to Differences in Age?}

At a fixed chemical composition, an older cluster has a bluer HB morphology. The RR Lyrae variables in an older

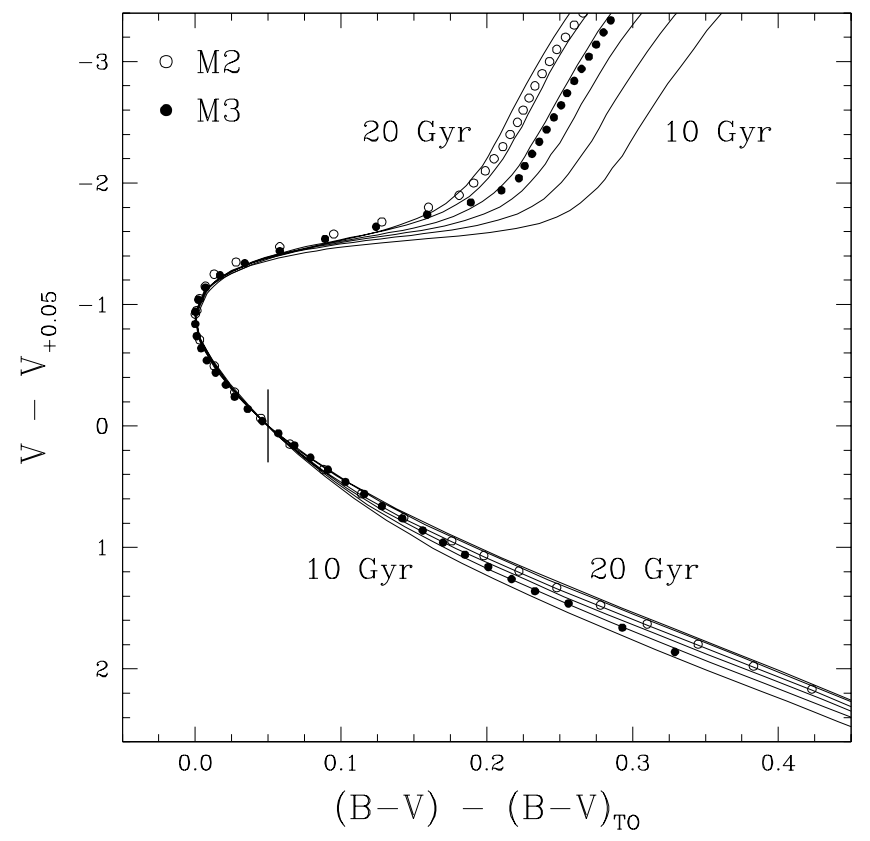

FIG. 7.-Plot of $(B-V)-(B-V)_{\mathrm{TO}}$ vs. $V-V_{+0.05}$ for $\mathrm{M} 2$ and M3. The M2 fiducial sequence is represented by open circles and the M3 fiducial sequence by filled circles. The model 1996 Yale isochrones $^{4}$ for $Z=0.0004$ and $Y=0.23$ with ages ranging from 10 to $20 \mathrm{Gyr}$ are also represented by solid lines. The age difference between M2 and M3 is about 2 Gyr, in the sense that M2 is older.

BHB cluster would then be more evolved and hence have faster period changes and higher luminosities, as observed for M2 compared with M3. The synthetic HB model calculations of Lee, Demarque, \& Zinn (1994, hereafter LDZ94) predict that an age difference of 2-3 Gyr, in the sense that M2 is older than M3, would suffice to explain the differences in their HB morphologies.

We explore the difference in age using, in essence, color differences between the color of the base of the RGB and that of the turnoff. VandenBerg, Bolte, \& Stetson (1990) described a method to derive accurate relative cluster ages with similar chemical compositions. They recommended shifting the clusters' CMDs in color until the turnoff colors agreed, then shifting the magnitudes until they matched at a point $0.05 \mathrm{mag}$ redder than the turnoff, $V_{+0.05}$. The color differences of the resulting RGBs could then be used to estimate the relative ages. The color difference between the turnoff and the giant branch is a monotonic and inverse function of age. Further, this color difference is independent of distance, reddening, and photometric zero-point errors. The method appears to be nearly independent of metallicity, particularly for $[\mathrm{Fe} / \mathrm{H}]<-1.2$, although there is some sensitivity to differences in elemental abundance ratios.

In Figure 7, we present the plot of $(B-V)-(B-V)_{\mathrm{TO}}$ versus $V-V_{+0.05}$ for M2 and M3. We also present the model 1996 Yale isochrones ${ }^{4}$ for $Z=0.0004$ and $Y=0.23$ $([\mathrm{Fe} / \mathrm{H}] \approx-1.66)$ with the ages ranging from 10 to $20 \mathrm{Gyr}$. Our results indicate that M2 is about 2 Gyr older than M3.

\footnotetext{
${ }^{4}$ See Demarque, P., Chaboyer, B., Guenther, D., Pinsonneault, M, Pinsonneault, L., \& Yi, S., Yale Isochrones 1996, at http:// shemesh.gsfc.nasa.gov/iso.html.
} 
Assuming that the two sets of photometry are accurate and that our smoothing technique has not introduced systematic effects, it becomes clear that the age difference is responsible for the RR Lyrae luminosity differences between M2 and M3. (We note, however, that Stetson, VandenBerg, \& Bolte 1996 have claimed that deep CMD data they obtained for both M2 and M3 indicates that the clusters do not differ in age. We await final presentation of their data and results with keen interest.) The blue HB morphology of M2 is due to its older age and, as a consequence, the M2 RR Lyrae variables are evolving away from their ZAHB locations and so are more luminous than those in M3. The more rapid mean period change rate of M2 RR Lyrae variables strongly supports this result.

We note that the synthetic HB morphology calculations of LDZ94 indicated a larger age difference than we have obtained. We address this issue in Appendix A.

\subsection{Do the Oosterhoff Classes Differ in Origin?}

We ask this question because it appears that the Oosterhoff dichotomy may be caused by differences in age, even when the clusters' metallicities are similar. One explanation is that the Oosterhoff I and II clusters all formed as part of the Galaxy's early evolution but that, perhaps, there was a hiatus in star formation that lasted 1-2 Gyr and occurred when the mean metallicity level reached a value of $[\mathrm{Fe} /$ $\mathrm{H}]=-1.5$ or so. Another explanation may be that the Galaxy accreted some of its globular clusters from small "proto-Galactic fragments" as described by Searle \& Zinn (1978) or from dwarf galaxies. Here we explore data relevant to the possible differences in origin between the Oosterhoff I and Oosterhoff II clusters.

Rodgers \& Paltoglou (1984) argued that clusters with $-1.3>[\mathrm{Fe} / \mathrm{H}]>-1.7$ have retrograde rotation about the Galaxy, while the other metallicity groups are rotating more slowly but in the same sense as the stars in the solar neighborhood. It is interesting that this is the metallicity region of most Oosterhoff I clusters. They also noticed that retrograde rotation may be related to the HB morphology. They claimed that high retrograde rotation clusters appear to have a more restricted range of HB morphologies, with Mironov HB color distribution index of $B / B+$ $R=0.48 \pm 0.19$, than do all other clusters with similar metal abundances. Further, van den Bergh (1993a, 1993b) argued that the Oosterhoff I clusters lie on retrograde orbits more frequently than do Oosterhoff II clusters. Finally, van den Bergh (1993b) suggested that orbit shape and Galacto-

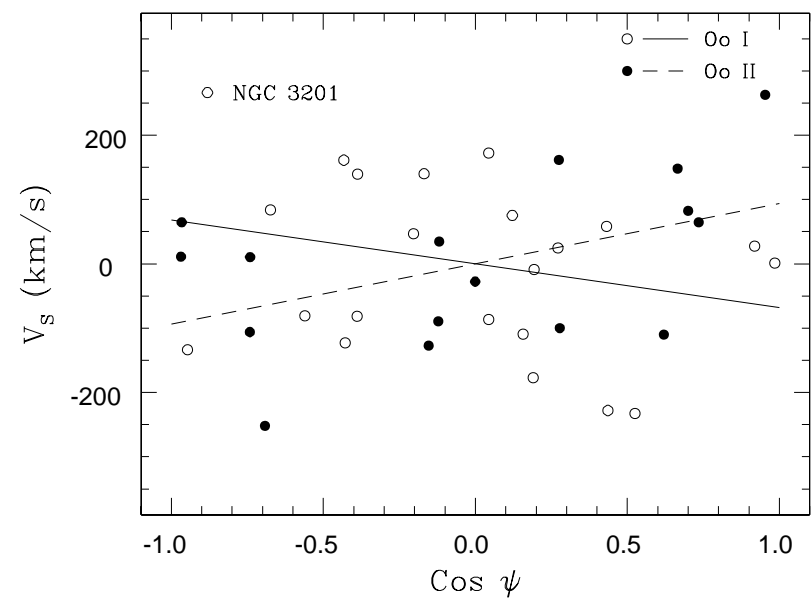

FIG. 8.-Plot of $V_{S}$ vs. $\cos \psi$ for Oosterhoff group I and II clusters. The Oosterhoff group I clusters are represented by open circles and the Oosterhoff group II clusters by filled circles. The fitted lines indicate that the Oosterhoff group I clusters have retrograde rotation with a slightly higher line-of-sight velocity dispersion, while the Oosterhoff group II clusters have prograde rotation with a smaller line-of-sight velocity dispersion.

centric distance are correlated with a tendency for clusters at large distance to have plunging orbits and below-average integrated luminosities. He suggested that the Oosterhoff group II clusters were formed very early in the evolutionary history of the proto-Galaxy and Oosterhoff group I clusters were formed at somewhat later date, consistent with our derived greater age for M2 compared with M3.

In Figure 8, we present a plot of $V_{S}$ versus $\cos \psi$ for the Oosterhoff I and II clusters (see Table 7) using the relations given by Frenk \& White (1980) and corrected by Zinn (1985), where $V_{S}$ is the radial velocity observed at the Sun's position by an observer at rest with respect to the galactic center and $\psi$ is the angle between the line of sight and the direction of galactic rotation at the cluster (Zinn 1993). The mean rotation velocity $\left\langle v_{\text {rot }}\right\rangle$ is given by the slope of the straight line, and the line-of-sight velocity dispersion $\sigma_{\text {los }}$ is given by the standard deviation of the points about the line. The fit for the Oosterhoff I clusters is represented by the solid line, and that for the Oosterhoff II clusters by the dashed line in Figure 8. The Oosterhoff I clusters appear to have zero or even retrograde rotation with $\left\langle v_{\text {rot }}\right\rangle=-68$ $\pm 56 \mathrm{~km} \mathrm{~s}^{-1}$ and $\sigma_{\mathrm{los}}=131 \pm 28 \mathrm{~km} \mathrm{~s}^{-1}$, while the Oosterhoff II clusters have prograde rotation with $\left\langle v_{\text {rot }}\right\rangle=+94$

TABLE 7

OOSTERHOFF I AND II CluSTERS

\begin{tabular}{|c|c|}
\hline Type & Cluster \\
\hline Oosterhoff I....... & 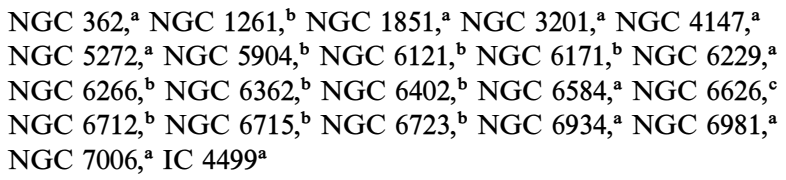 \\
\hline Oosterhoff II...... & 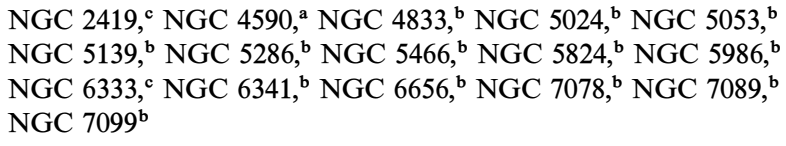 \\
\hline
\end{tabular}

${ }^{a}$ Young halo group clusters.

${ }^{b}$ Old halo group clusters.

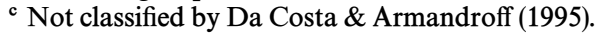


$\pm 47 \mathrm{~km} \mathrm{~s}^{-1}$ and $\sigma_{\text {los }}=115 \pm 29 \mathrm{~km} \mathrm{~s}^{-1}$, confirming the conclusions of Rodgers \& Paltoglou (1984) and van den Bergh (1993a). If NGC 3201, which has a very large radial velocity, is excluded from Oosterhoff I sample, $\left\langle v_{\text {rot }}\right\rangle=$ $-31 \pm 56 \mathrm{~km} \mathrm{~s}^{-1}$ and $\sigma_{\mathrm{los}}=124 \pm 27 \mathrm{~km} \mathrm{~s}^{-1}$, and the Oosterhoff group I clusters still differ from the results for the Oosterhoff group II clusters. And removing a single cluster may introduce a bias, of course.

The different kinematics between Oosterhoff I and II clusters hints that they might have experienced different formation histories, because it is hard to reconcile the evolution of an older, more metal-poor system into a younger, more metal-rich one which has a slower, possibly even retrograde net Galactic rotation as well as a slightly higher line-of-sight velocity dispersion. The dichotomy is reminiscent of the "young halo" versus "old halo" model of Zinn (1993) and Da Costa \& Armandroff (1995). Recall that "young halo" clusters were distinguished from "old halo" clusters by having unusually red HB colors at fixed metallicity. Da Costa \& Armandroff (1995) found $\left\langle v_{\text {rot }}\right\rangle=-46$ $\pm 81 \mathrm{~km} \mathrm{~s}^{-1}$ and $\sigma_{\text {los }}=163 \pm 25 \mathrm{~km} \mathrm{~s}^{-1}$ for the "young halo" clusters and $\left\langle v_{\text {rot }}\right\rangle=+50 \pm 27 \mathrm{~km} \mathrm{~s}^{-1}$ and $\sigma_{\text {los }}=$ $106 \pm 11 \mathrm{~km} \mathrm{~s}^{-1}$ for the "old halo" clusters.

We are left with the impression that the Oosterhoff I and II clusters may have had independent origins and that perhaps the latter, given their prograde rotation, are likely to have been more closely coupled to the formation of the Galaxy. The Oosterhoff I clusters could have formed more slowly in smaller proto-Galactic fragments with little or no net angular momentum (in the Galactic frame) that later merged with the Galaxy. The conclusion is not compelling, of course, since the number of clusters in our sample is small and the uncertainties in net rotation are, in consequence, large. Further, we wonder if there is a population of clusters with higher metallicities than the Oosterhoff II clusters whose kinematics are also prograde. If these are not the Oosterhoff I clusters, then where are they? Are they a subset of the Da Costa \& Armandroff "old clusters"?

\subsection{Field RR Lyrae Variables}

If the Oosterhoff I and II clusters have different kinematics, they should also have different spatial distributions. Unfortunately, the number of clusters available for study is small, and the spatial distribution of clusters is a function of both their origins and their destruction due to tidal forces. Field RR Lyrae variables offer us another opportunity to explore the relations between periods, spatial distributions, kinematics, and metallicities. We note at the outset that the field RR Lyrae variables also manifest the Oosterhoff dichotomy (Preston 1959; Suntzeff, Kinman, \& Kraft 1991).

Several relevant studies of field RR Lyrae variables and HB stars have already been done. Preston, Schectman, \& Beers (1991) and Kinman, Suntzeff, \& Kraft (1994) argued that the field HB morphology in the solar neighborhood is considerably bluer than that farther from the plane, indicating that such differences might be a consequence of differences in the mean ages of the field HB populations in the sense that field HB stars near the plane are older. Wilhelm et al. (1996) studied 525 field horizontal-branch stars in the Galactic halo, and found results rather similar to those above. Restricting the analysis to stars with $[\mathrm{Fe} /$ $\mathrm{H}] \leq-1.6$, they found that stars within $4 \mathrm{kpc}$ of the plane showed a Galactic rotational velocity of $+40 \pm 17 \mathrm{~km} \mathrm{~s}^{-1}$, while those more than $4 \mathrm{kpc}$ from the plane are in retro- grade rotation, $\left\langle v_{\text {rot }}\right\rangle=-93 \pm 36 \mathrm{~km} \mathrm{~s}^{-1}$. Layden (1996) presented a preliminary analysis of the kinematics of $\mathrm{RR}$ Lyrae variables near and far from the plane and confirmed the apparent change from prograde to retrograde rotation.

We restudy the question from a slightly different perspective, asking whether the period distributions of $R R$ Lyrae variables also appear to be a function of distance from the Galactic plane. At what distance from the plane should we expect to see differences between the Oosterhoff I and II variables? A clue is provided by the results discussed above, and those of Majewski (1992), who found that metalpoor field stars that lie more than $5 \mathrm{kpc}$ from the plane appear to have net retrograde rotation. Carney et al. (1996) also found net retrograde rotation for metal-poor field stars whose orbits carry them more than $5 \mathrm{kpc}$ from the plane. We therefore concentrate on stars about $5 \mathrm{kpc}$ or more from the plane versus those closer to the plane. (We must recognize, of course, that if two populations or histories exist, the RR Lyrae variables at any distance from the plane are an admixture of both populations. We seek only evidence that there may be a change in the dominance of one population versus another as a function of distance from the plane.)

To obtain a sample of RR Lyrae variables for study, we must be very careful to avoid systematic selection effects. There must not be any bias, or at least very little bias, in metallicities or pulsational periods for the stars. We therefore used the unbiased sample of R Rab variables discovered at Lick Observatory and at Palomar Observatory (Preston 1959; Butler et al. 1982; Kinman, Mahaffey, \& Wirtanen 1982, Kinman, Suntzeff, \& Kraft 1984, Kinman et al. 1985; Suntzeff et al. 1991; Suntzeff, Kraft, \& Kinman 1994, hereafter SKK94; Kinman 1998, private communication; Suntzeff 1998, private communication) and by Saha (1984) and Saha \& Oke (1984). The metallicities of the RR $a b$ variables were estimated from $\Delta S$ measurements using the relation of SKK94

$$
[\mathrm{Fe} / \mathrm{H}]=-0.158 \Delta S(\mathrm{RR} a b)-0.408 .
$$

The values of $[\mathrm{Fe} / \mathrm{H}]$ are on the Zinn \& West (1984) scale of metallicities for globular clusters. The $\Delta S$ measurements of Preston (1959) appear to be systematically different from those given by SKK94, and we therefore used metallicities given by them for 63 common variables. To estimate metallicities for variables from Preston (1959), we derived a linear relation between $\Delta S$ measurements of SKK94 and those of Preston (1959) using common variables

$$
\Delta S(\mathrm{RR} a b)_{\mathrm{SKK} 94}=0.892 \Delta S(\mathrm{RR} a b)_{\text {Preston }}+0.965,
$$

and then we obtained metallicities by applying equation (7).

The distances were estimated using mean magnitudes of RR Lyrae variables. However, because of uncertainties involved in the mean magnitudes of the field RR Lyrae variables and the interstellar reddening, we choose not to divide the field RR Lyrae variables at a single distance from the plane. Instead, we divide the samples into those closer to the plane $(|Z| \leq 3 \mathrm{kpc}, 125$ variables), which may be expected to be related to the formation of the Galaxy, and those in the halo $(|Z| \geq 5 \mathrm{kpc}, 61$ variables), which may be more likely to include stars gathered to the Galaxy via accretion.

In Figure 9, we present the metallicity distributions of the field RR Lyrae variables. The peaks in the metallicity distribution occur at $[\mathrm{Fe} / \mathrm{H}]=-1.60,-1.30$, and -0.60 for 


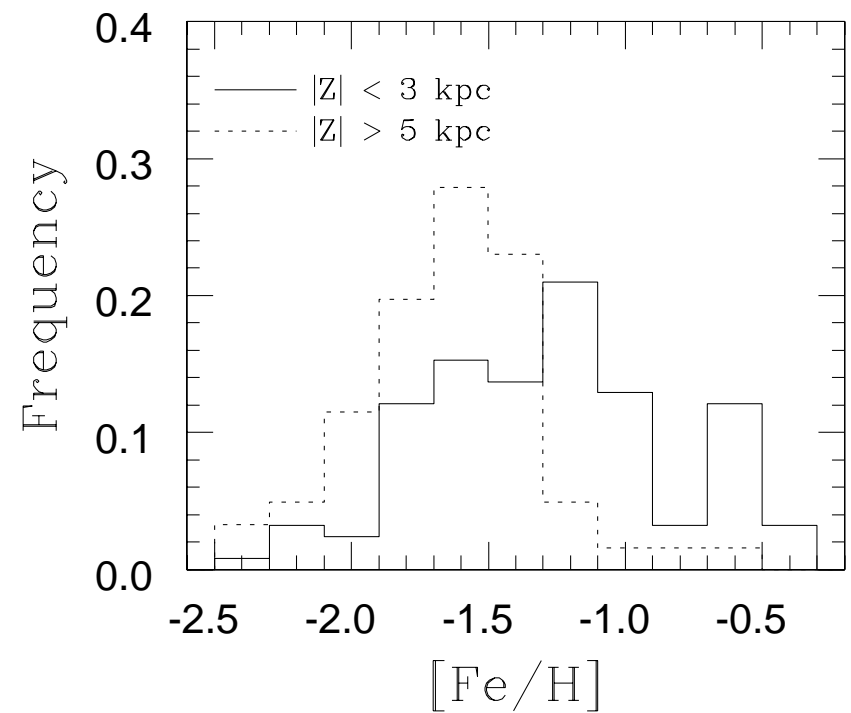

FIG. 9.-Metallicity distributions for an unbiased sample of field RR $a b$ variables discovered at Lick Observatory and by Saha (1984). The solid line represents RR $a b$ variables with $|Z| \leq 3 \mathrm{kpc}$, and the dotted line represents RR $a b$ variables with $|Z| \geq 5 \mathrm{kpc}$.

RR Lyrae variables with $|Z| \leq 3 \mathrm{kpc}$ and at $[\mathrm{Fe} /$ $\mathrm{H}]=-1.60$ for those with $|Z| \geq 5 \mathrm{kpc}$. We performed Kolmogorov-Smirnov (K-S) tests to see if their metallicity distributions are drawn from the same parent population. Our results show that the probability of being drawn from identical populations is $0.00009 \%$ with a K-S discrepancy of 0.422 , indicating that they have different parent populations. Of course, this more likely indicates the presence of yet another population of RR Lyrae variables, with metallicities typical of the thick disk population $(\langle[\mathrm{Fe} /$ $\mathrm{H}]\rangle \approx-0.6$ ), which appear in the sample with $|Z| \leq 3$ $\mathrm{kpc}$. If we restrict the comparison to variables with $[\mathrm{Fe} / \mathrm{H}]$ $\leq-0.8$, the K-S test indicates a probability of $0.005 \%$ that the variables are drawn from the same parent population. The two samples of variables appear to have different metallicities. How about their periods?

We present the period distributions of the two samples in Figure $10 a$. One interesting feature in Figure $10 a$ is the strong peak in RR $a b$ variables with $P_{0}=0.55$ day, indicative of Oosterhoff $\mathrm{I}$, and the absence of variables at $P_{0}=$ 0.65 day, indicative of Oosterhoff II, in the sample population with $|Z| \geq 5 \mathrm{kpc}$. This is consistent with the $\mathrm{RR}$ Lyrae variables far from the Galactic plane being dominated by the Oosterhoff I class. If the RR Lyrae population farther from the plane is dominated by the Oosterhoff I class, then is the RR Lyrae population closer to the plane dominated by the Oosterhoff II field counterpart? Compared to the RR Lyrae population with $|Z| \geq 5 \mathrm{kpc}$, the RR Lyrae population with $|Z| \leq 3 \mathrm{kpc}$ has several peaks and a wider range in the period distribution. This makes it harder to discern a clear Oosterhoff I versus II dominance. Some of this is due to contamination by the more metalrich, thick disk variables, so in Figures $10 b$ and $10 c$ we show the period distributions for $[\mathrm{Fe} / \mathrm{H}] \leq-0.8$, as above, and also for $[\mathrm{Fe} / \mathrm{H}] \leq-1.6$. The latter regime corresponds more closely to the metallicities of the Oosterhoff II clusters and so should help reveal them in the field populations. The $\mathrm{K}-\mathrm{S}$ tests show that for $[\mathrm{Fe} / \mathrm{H}] \leq-0.8$ and $[\mathrm{Fe} /$ $\mathrm{H}] \leq-1.6$, the probabilities that two samples are drawn

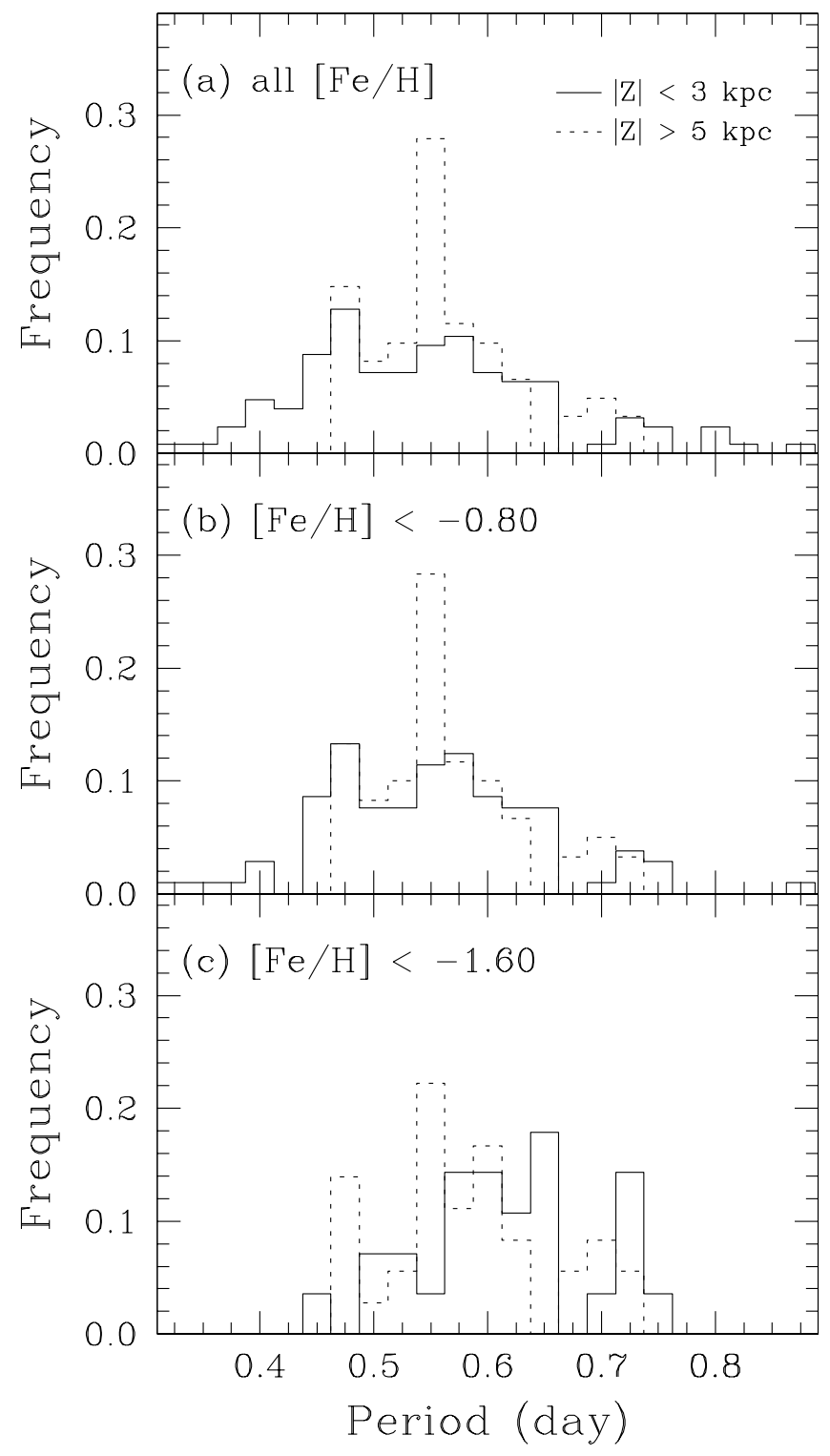

FIG. 10.-Period distributions for an unbiased sample of field RR $a b$ variables with different metallicity ranges. The solid lines represent RR $a b$ variables with $|Z| \leq 3 \mathrm{kpc}$, and the dotted lines represent $\mathrm{RR} a b$ variables with $|Z| \geq 5 \mathrm{kpc}$. The K-S tests (Table 8) indicate that they belong to different populations in terms of period distribution. Note the absence of variables at $P_{0}=0.65$ day in the RR Lyrae sample with $|Z| \geq 5 \mathrm{kpc}$.

from the identical parent populations are $19.9 \%$ and $15.3 \%$, respectively (see Table 8). Indeed, in Figure 10c, one may see that the reason for the poor agreement is the peak near 0.55 day for the variables with $|Z| \geq 5 \mathrm{kpc}$ and the peak near 0.65 day for those with $|Z| \leq 3 \mathrm{kpc}$.

This result, however, may appear to be inconsistent with that of Catellani, Maceroni, \& Tosi (1983), whose result implies that the Oosterhoff II class would be dominant at higher distances from the plane. We address this issue in Appendix B.

Thus field star results suggest that the Oosterhoff II variables are confined more closely to the plane, while the cluster results suggest that they have prograde rotation. The Oosterhoff I variables show zero or retrograde rotation and lie, on average, farther from the plane. This agrees with Layden's (1996) analyses of the differences in net rotation as 
TABLE 8

Results of Kolmogorov-Smirnov TeStS

\begin{tabular}{ccccc}
\hline \hline$[\mathrm{Fe} / \mathrm{H}]$ & $n(|Z| \leq 3 \mathrm{kpc})$ & $n(|Z| \geq 5 \mathrm{kpc})$ & $\begin{array}{c}\text { Probability } \\
(\%)\end{array}$ & K-S Discrepancy \\
\hline All $\ldots \ldots \ldots \ldots$ & 125 & 61 & 1.81 & 0.240 \\
$\leq-0.80 \ldots \ldots$ & 105 & 60 & 19.9 & 0.174 \\
$\leq-1.60 \ldots \ldots$ & 28 & 36 & 15.3 & 0.286 \\
\hline
\end{tabular}

NoTE.-Measuring period distributions between RR Lyrae populations with $|Z| \leq 3 \mathrm{kpc}$ and $|Z| \geq 5 \mathrm{kpc}$.

a function of distance from the plane for RR Lyrae variables and those of Wilhelm et al. (1996) for horizontal-branch stars in general. Given the bluer HBs of Oosterhoff II clusters, this is also consistent with the results of Preston et al. (1991) and Kinman et al. (1994) that the mean color of the HB is bluer near the plane than farther away.

\section{CONCLUSIONS}

We have discussed the luminosity differences between the Oosterhoff II cluster M2 and the Oosterhoff I cluster M3. Both the period shift analysis and the main-sequence fitting show that the M2 RR $a b$ variables are about 0.2 mag brighter than M3 RR $a b$ variables. Comparisons with Oosterhoff I clusters, NGC 3201 and NGC 7006, also imply that the M2 RR Lyrae variables are more luminous. On the other hand, the period shift analysis indicates that the M2 variables have similar luminosities to those in the Oosterhoff II cluster NGC 5986. The luminosity difference is probably caused by the evolutionary effect as claimed by LDZ90: the M2 RR $a b$ variables have evolved away from the ZAHB while the M3 RR $a b$ variables are closer to the ZAHB. A comparison of the mean period change rates of two clusters supports this hypothesis.

The differences in the evolutionary stages of the M2 and M3 RR Lyrae variables are probably caused by differences in cluster ages. Our relative age estimation using the difference in color between the base of giant branch and mainsequence turnoff shows that M2 is about 2 Gyr older than M3.

The kinematic differences between Oosterhoff group I and II clusters indicate that they may have different origins. The zero or retrograde net rotation with a slightly higher velocity dispersion of the Oosterhoff group I clusters suggests that at least some of them joined the Galaxy via accretion. Our result of a $2 \mathrm{Gyr}$ age difference between the
Oosterhoff II cluster M2 and Oosterhoff I cluster M3 hints that the Oosterhoff II clusters were formed very early in the proto-Galaxy and the Oosterhoff I clusters formed later. The higher metallicities and younger ages of the Oosterhoff I clusters are consistent with a model of coherent evolution of the Galaxy, but the slower rotation of the these clusters relative to the older and more metal-poor Oosterhoff II clusters is not consistent with such a model.

The period distributions of an unbiased sample of field $\mathrm{RR} a b$ variables with $|\mathrm{Z}| \leq 3 \mathrm{kpc}$ and $|Z| \geq 5 \mathrm{kpc}$ suggest that the (probably) older, more metal-poor, and longer period Oosterhoff II variables are confined more closely to the plane than are the (probably) younger, more metal-rich, and shorter period Oosterhoff I variables. This is also unexpected in a coherent-evolution model of the Galaxy.

We have shown that the generally accepted Gaussian HB mass-dispersion rate (i.e., the mass-loss rate at RGB tip) in synthetic HB model calculations cannot fully explain the extended blue HB population and the pulsational properties of RR Lyrae variables in M2. Comparisons with synthetic HB models strongly suggest that an enhanced mass-loss mechanism is required. We also have discussed the statistical effect on the metallicity estimate using $P_{0 \text {,min }}$ for the field RR $a b$ variables. Our calculations suggest that the statistical effect is sufficient to explain the apparent gradient in $P_{0, \min }$ without introducing a metallicity effect.

We thank T. Kinman and N. Suntzeff for providing us invaluable Lick RR Lyrae data before publication. We thank an anonymous referee for useful comments and a careful review of the paper. We also thank the National Science Foundation for financial support via grants AST 96-19381 and AST 98-0531 to the University of North Carolina. We also made very profitable use of the SIMBAD database, operated at CDS, Strasbourg, France.

\section{APPENDIX A}

\section{THE EFFECTS OF MASS-LOSS DISPERSION ON HB MORPHOLOGY AND AGE ESTIMATES}

We present the M2 HB region CMD in Figure $11 a$ and the $l_{\mathrm{HB}}$ distribution in Figure $11 b$ (see $\S 4.3$ of LC99 for details). The parameter $l_{\mathrm{HB}}$ is designed to quantify position along the $\mathrm{HB}$, especially the blue sections, relative to some fiducial point. We adopt the $l_{\mathrm{HB}}$ length scaling factors given by Catelan et al. (1998) and M. Catelan (1998, private communication), with $\Delta l_{\mathrm{HB}} / \Delta(B-V)_{0}=22.24$ and $\Delta l_{\mathrm{HB}} / \Delta M_{V}=4.63$. As kindly noted by M. Catelan (1998, private communication), these are the same relations obtained by Dixon et al. (1996) for M79. The M2 HB fiducial line was converted into the $\left(l_{\mathrm{HB} x}, l_{\mathrm{HB} y}\right)$-plane and the total length was integrated along the converted fiducial line. Each HB star was then projected onto that plane and their $l_{\mathrm{HB}}$ coordinates were computed perpendicular to the converted fiducial line. This $l_{\mathrm{HB}}$ coordinate system effectively removes the degeneracy in HB $B V$ colors for $(B-V)_{0}<0$ and is very useful for studying the presence of gaps on the HB distribution.

We performed a Monte Carlo simulation to reproduce the observed M2 HB distribution and its CMD using the HB evolutionary tracks of Yi et al. (1997) for $Z=0.0004$ and $Y=0.24$. We employed the modified Gaussian HB mass distribution relation of LDZ90. We constructed more than 500 synthetic HB models and each model has $262 \pm 3$ stars, which is 


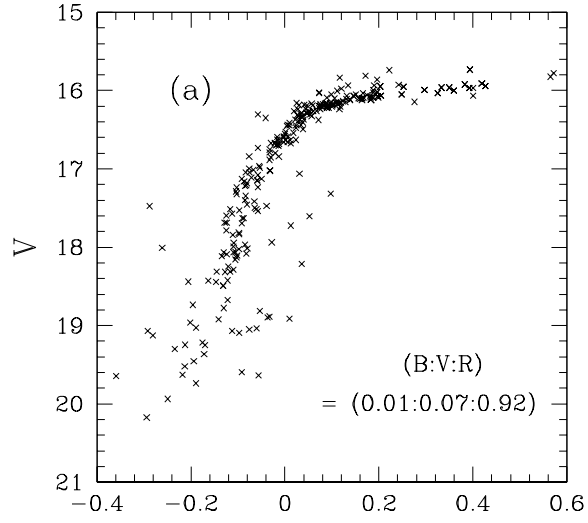

$(\mathrm{B}-\mathrm{V})$

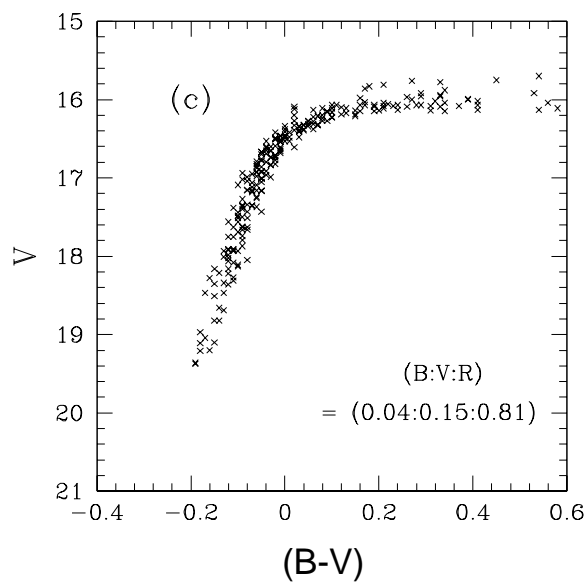

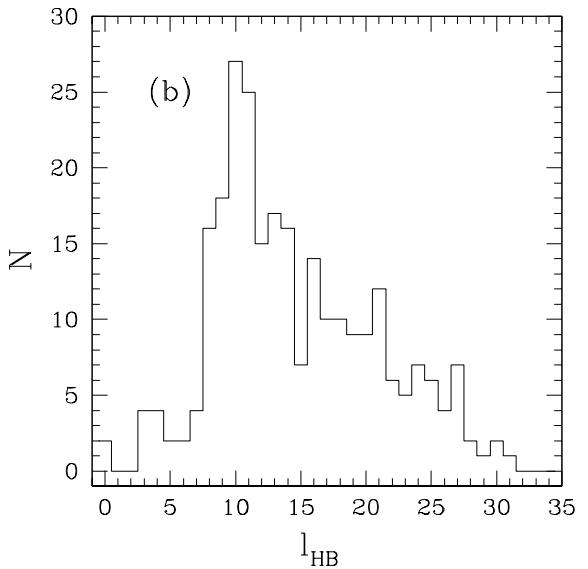

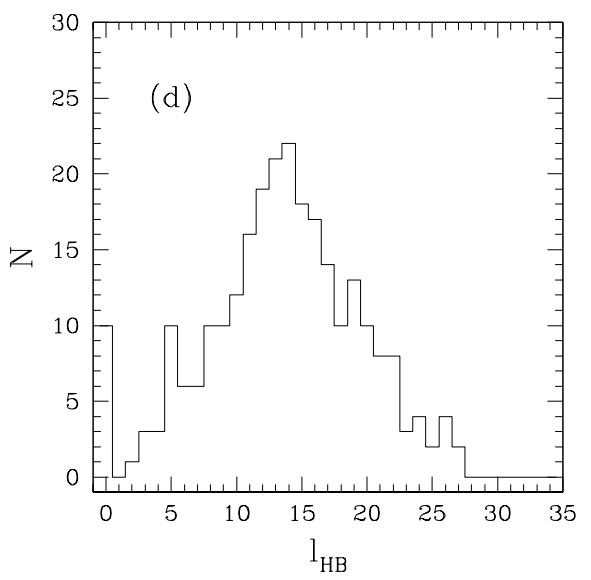

FIG. 11.-(a) Observed HB region CMD for M2. (b) Observed M2 $l_{\mathrm{HB}}$ distribution. (c) Synthetic HB CMD that best matches the observed distribution, obtained from K-S tests. (d) Synthetic $l_{\mathrm{HB}}$ distribution.

similar to the number of HB stars in our CMD. The HB mean mass $\left\langle M_{\mathrm{HB}}\right\rangle$ was varied from 0.58 to $0.68 M_{\odot}$ and the HB mass-dispersion rate $\sigma\left(M_{\mathrm{HB}}\right)$ was varied from 0.030 to $0.090 M_{\odot}$. We performed K-S tests of the synthetic $l_{\mathrm{HB}}$ distribution for each model with respect to the observed $l_{\mathrm{HB}}$ in M2. In Figures $11 c$ and $11 d$, we present the synthetic CMD and $l_{\mathrm{HB}}$ distribution of the model simulation that best matched the observed one. It has $\left\langle M_{\mathrm{HB}}\right\rangle=0.64 M_{\odot}$ and $\sigma\left(M_{\mathrm{HB}}\right)=0.087 M_{\odot}$. Even in this best case, the single Gaussian HB mass distribution failed to reproduce the observed M2 HB morphology.

We also present the $l_{\mathrm{HB}}$ distribution of the HB evolutionary tracks of Yi et al. (1997) for $Z=0.0004$ and $Y=0.24$ in Figure 12. It should be noted that the locations of the ZAHB in the $l_{\mathrm{HB}}$ coordinate are rather evenly spaced in the HB mass unit. The figure shows that the HB stars remain near their ZAHB positions for the most of their lifetimes.

We compared the skewness (the $3^{\text {rd }}$ moment) of the observed $l_{\mathrm{HB}}$ distribution to those of the synthetic $\mathrm{HB}$ models described above. The skewness of the distribution characterizes the degree of asymmetry of a distribution around its mean. A positive value of skewness signifies a distribution with an asymmetric tail extending out toward more positive $x$-axis; a negative value signifies a distribution whose tail extends out toward more negative $x$-axis (Press et al. 1992). The skewness of the observed $\mathrm{M} 2 l_{\mathrm{HB}}$ distribution is $+0.45 \pm 0.24$ (the error is the standard deviation) and is significantly higher than those of synthetic $\mathrm{HB}$ model simulations, $-0.41 \pm 0.38$. The negative value of the skewness of synthetic HB model calculations is thought to be the effect of the HB evolution as shown in Figure 12. The positive skewness of the observed $\mathrm{M} 2 l_{\mathrm{HB}}$ distribution is nothing but a quantitative presentation of a tail extending out toward more positive $l_{\mathrm{HB}}$ coordinate (i.e., toward lower HB mass and hotter ZAHB temperatures) as already shown in Figure 11b, which does not occur with a single Gaussian HB mass distribution. The positive skewness may indicate that there is a non-Gaussian or enhanced mass-loss mechanism. During red giant evolution, enhanced mass loss could populate the extremely blue sections of M2's HB. Presumably, this very blue extension of M2's CMD would be in addition to that caused by the cluster's greater age compared to M3. The difference between our estimate of the variation in age in M2 and M3, 2 Gyr, compared to that estimated by LDZ94, 3 Gyr, may be attributed to this enhanced mass-loss mechanism. To produce a blue HB morphology with a single Gaussian mass-dispersion rate, such as given by LDZ90, requires a shift of a mean HB mass toward a lower mass scale (i.e., it requires a greater age). However, if a non-Gaussian (or a Gaussian plus an additional decreasing HB mass distribution function with decreasing HB mass) HB mass distribution is adopted, then blue HB stars would be more efficiently produced, and it may reduce ages derived from synthetic HB models that assume a simple Gaussian HB mass distribution. 


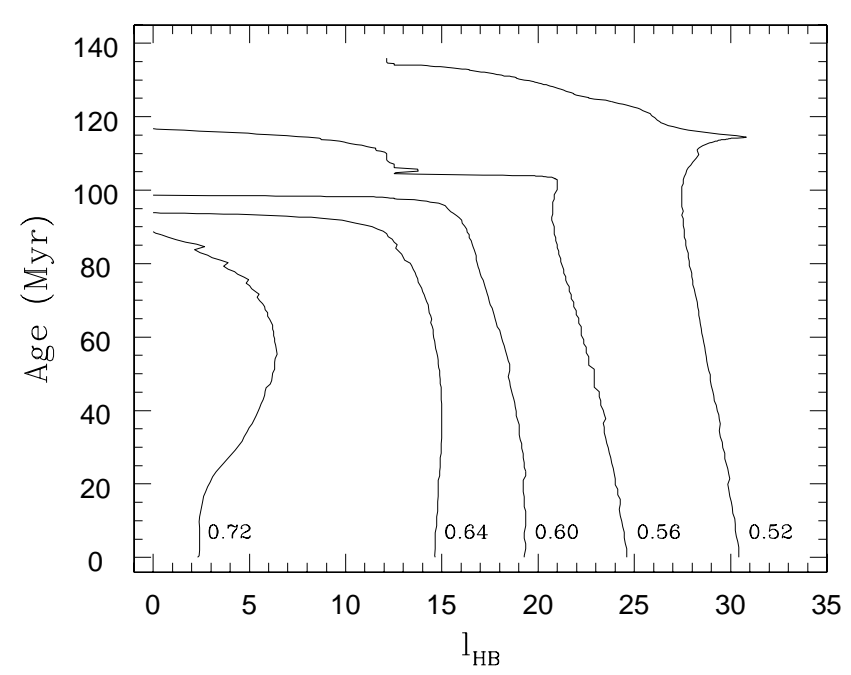

FIG. 12. $-l_{\mathrm{HB}}$ coordinates vs. evolutionary ages for theoretical HB tracks of Yi et al. (1997) for $Z=0.0004$ and $Y=0.24$. The masses of the HB tracks are also indicated.

A comparison with the synthetic HB calculations for period shift determinations of Lee (1990) also hints at a non-Gaussian HB mass distribution. He presented the synthetic “reduced" period shift $\Delta \log P_{383}^{\prime}=\log P_{\text {cluster }}^{\prime}-\log P_{\mathrm{M} 3}^{\prime}$ at $\log T_{\text {eff }}=$ 3.83, where $\log P^{\prime}=\log P_{0}+0.336\left(M_{\mathrm{bol}}-\left\langle M_{\mathrm{bol}}\right\rangle\right)$ in his Figures $3 a$ and $3 b$. Since the bolometric corrections for M2 and M3 RR $a b$ variables are expected to be similar, as we discussed in $\S 4.1$, we used the intensity-weighted integrated $V$ magnitudes for our calculations of the $M_{\text {bol }}$. Using equation (6) above, we calculated the reduced period shift of M2 with respect to M3, $\Delta \log P_{383}^{\prime}=0.0766 \pm 0.0085$, and this value is similar to the period shift between M2 and M3 that we have obtained in $\S 4.1$, $\Delta \log P_{0}=0.0771$. At $(B-R) /(B+V+R)=0.92$, Lee's $(1990)$ calculations predict $\Delta \log P_{383}^{\prime} \approx 0.075$ for $\sigma\left(M_{\mathrm{HB}}\right)=0.01$ $M_{\odot}$ and 0.030 for $\sigma\left(M_{\mathrm{HB}}\right)=0.03 M_{\odot}$. The synthetic HB calculations for period shift determinations using a Gaussian HB mass distribution require a significantly smaller HB mass dispersion to match the observed period shift in M2 relative to M3, and it is not consistent with our synthetic HB model simulations above, which require an HB mass dispersion of at least $\sigma\left(M_{\mathrm{HB}}\right) \approx 0.08 M_{\odot}$ to reproduce the observed total $l_{\mathrm{HB}}$ length of M2. Hence, to explain the observed HB morphology and the pulsational properties of RR Lyrae variables in M2, a sharply peaked (perhaps Gaussian) distribution at $l_{\mathrm{HB}} \approx 10$ and an additional extended non-Gaussian distribution for $l_{\mathrm{HB}} \geq 15$ in our Figure $12 b$ are required. Finally, understanding the proper mass-loss mechanism may provide an amelioration of the problem, such as can be seen in M15 (see, e.g., Rood 1990 for details).

\section{APPENDIX B}

\section{THE STATISTICAL EFFECT ON THE METALLICITY ESTIMATE USING $P_{0, \min }$}

Castellani et al. (1983) argued that the minimum period of field RRab variables $P_{0, \min }$, appears to be correlated with the distance from the Galactic plane in the sense that $P_{0, \min }$ increases with the distance from the plane. Using very heterogeneous data (see references therein), they found that $\Delta \log P_{0, \min } / \Delta|Z|=+0.004 \mathrm{kpc}^{-1}$. They noted that the $P_{0, \min }$ of globular clusters appears to be correlated with metallicities (e.g., see Fig. 4 of Castellani \& Tornambe 1981). They argued that this gradient in the $P_{0, \min }$ is due to the difference in metallicities of field RR $a b$ variables and found $\Delta[\mathrm{Fe} / \mathrm{H}] / \Delta|Z|=-0.02$ dex $\mathrm{kpc}^{-1}$. Their result then implies that the Oosterhoff II class would be dominant at higher distances from the galactic plane if this gradient is caused exclusively by metallicity differences.

We suggest that the apparent increase in $P_{0, \min }$ as $|Z|$ increases is most likely a simple statistical effect. As $|Z|$ increases and stellar densities drop exponentially, there will be fewer stars to populate the long- and short-period tails in the distribution of fundamental mode periods. Thus an apparent gradient in $P_{0, \min }$ is expected from statistical probabilities alone.

We have performed a Monte Carlo simulation to test our idea. We assumed that the stellar number density of field HB stars follows a exponential law

$$
N(Z)=N_{0} e^{-Z / h_{Z}},
$$

where $N_{0}$ is the number of field HB stars at $Z=0$ and $h_{Z}$ is the scale height of field HB stars. We assumed $h_{Z}=4 \mathrm{kpc}$ for the Oosterhoff I class and $2 \mathrm{kpc}$ for the Oosterhoff II class since the Oosterhoff II class appears to be more confined to the plane, as discussed in $\S 5.4$. Then the number of RRab variables as a function of the distance from the plane, $N_{\mathrm{ab}}(Z)$, can be approximated by

$$
N_{\mathrm{ab}}(Z) \approx N(Z) f_{1}(\mathrm{RR}) f_{2}(\mathrm{ab})
$$




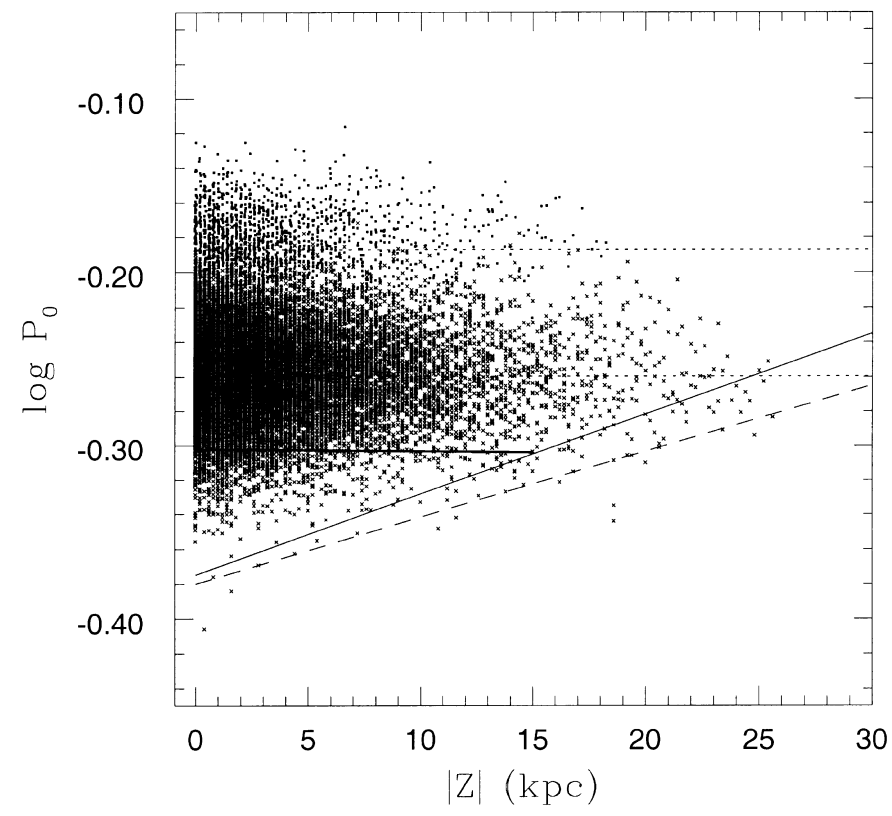

FIG. 13.-Plot of simulated $|Z|$ vs. $\log P_{0}$ for field RR $a b$ variables. The exponential stellar density law above the galactic plane $N(Z) \propto e^{-Z}$ is assumed. The total numbers of stars is 16,492 for Oosterhoff I class (crosses) and 1989 for Oosterhoff II class (dots). The mean periods for the Oosterhoff I and II are presented by dotted lines. The solid line represents the lower envelope of Castellani et al. (1983) with $\Delta \log P_{0, \min } / \Delta|Z|=+0.004 \mathrm{kpc}^{-1}$ and the dashed line presents the lower envelope of the Oosterhoff I class of this simulation with $\Delta \log P_{0, \min } / \Delta|z|=+0.0038 \mathrm{kpc}^{-1}$. The thick solid line represents $\Delta \log \left(\left\langle P_{0}\right\rangle-\mathrm{FWHM}\right) / \Delta|Z|$ with a slope of $-0.0001 \mathrm{kpc}^{-1}$.

where $f_{1}(\mathrm{RR})$ is the number ratio of $\mathrm{RR}$ Lyrae variables $(V)$ to the total number of HB stars $(B+V+R)$ and $f_{2}(\mathrm{ab})$ is the number ratio of RR $a b$ variables $n(\mathrm{ab})$, relative to the total number of RR Lyrae variables $n(\mathrm{ab}+\mathrm{c})$.

We constructed two synthetic models for the Oosterhoff I and II classes and each model had 50,000 HB stars. We adopted $f_{1}(\mathrm{RR})=0.40$ from the M3 data (Buonanno et al. 1994) and $f_{2}(\mathrm{ab})=0.83$ (Smith 1995) for the Oosterhoff I class, and for the Oosterhoff II class we adopted $f_{1}(\mathrm{RR})=0.07$ from our M2 data (LC99) and $f_{2}(\mathrm{ab})=0.56$ (Smith 1995). We calculated period distributions for each class as a function of the distance from the plane, using $\left\langle P_{0}\right\rangle=0.55 \pm 0.05$ day for the Oosterhoff I class and $0.65 \pm 0.05$ day for the Oosterhoff II class.

We present our calculations in Figure 13. The total numbers of RR $a b$ variables for the Oosterhoff I and II classes are 16,492 and 1989, respectively. In the figure, we present the lower envelope of Castellani et al. (1983) and that of our simulations found by eye. It should be noted that both fitted lines are somewhat subjective, but they have very similar slopes, $\Delta \log P_{0, \text { min }} / \Delta|Z|=+0.004 \mathrm{kpc}^{-1}$ (Castellani et al. 1983) and $+0.0038 \mathrm{kpc}^{-1}$. We also note that the lower envelope in our simulations is defined by the Oosterhoff I class only, unless we make $\sigma\left(P_{0}\right)$ for the Oosterhoff II class improbably large. The scale height for the Oosterhoff II class should not affect our $P_{0, \min }$ lines, and tests confirm this. We calculated the period distribution for the Oosterhoff II class using $h_{z}=4 \mathrm{kpc}$ and our $P_{0, \min }$ remains the same, unless we make $\sigma\left(P_{0}\right)$ for the Oosterhoff II class improbably large again. We performed a Gaussian fit with a binning size of $1 \mathrm{kpc}$ and calculated a FWHM for each bin up to $15 \mathrm{kpc}$. We also present $\Delta \log \left(\left\langle P_{0}\right\rangle-\mathrm{FWHM}\right) / \Delta|Z|$ in the figure. As expected, the slope of $\Delta \log \left(\left\langle P_{0}\right\rangle\right.$ $-\mathrm{FWHM}) / \Delta|Z|$ is negligibly small, $-0.0001 \mathrm{kpc}^{-1}$, since we assumed a constant $\sigma\left(P_{0}\right)$ for our calculations. The lower envelope $P_{0, \min }$ in our calculations, however, is defined by the short-period tail in the period distribution, which approaches to zero faster as the number of stars decreases (i.e., as the distance from the plane increases), and this statistical effect could produce a "pseudo"-gradient in the minimum period.

Our simulations suggest that the exponential drop in the number of stars with the distance from the plane could create an apparent gradient in $P_{0, \min }$ where none exists in the model $\left(\Delta \log \left(\left\langle P_{0}\right\rangle-\mathrm{FWHM}\right) / \Delta|Z|\right)$. A metallicity gradient therefore may not be required to explain the result of Castellani et al. (1983).

\section{REFERENCES}

Alcock, C., et al. 1996, AJ, 111, 1146

Alves, D. R., \& Sarajedini, A. 1999, ApJ, 511, 225

Bolte, M. 1992, ApJS, 82, 145

Bono, G., \& Castellani, V. 1992, A\&A, 258, 385

Buonanno, R., Crosi, C., Buzzoni, A., Cacciari, C., Ferraro, F. R., \& Fusi Pecci, F. 1994, A\&A, 290, 69

Buonanno, R., Crosi, C. E., Pulone, L., Fusi Pecci, R., \& Bellazzini, M. 1998, A\&A, 333, 505

Butler, D., Kemper, E., Kraft, R. P., \& Suntzeff, N. 1982, AJ, 87, 353

Buzzoni, A., Fusi Pecci, F., Buonnano, R., \& Corsi, C. E. 1983, A\&A, 128

Cacciari, C. 1984, AJ, 89, 231

Caputo, F., Roger, C. M., \& Paez, E. 1987, A\&A, 183, 228

Carney, B. W., Laird, J. B., Latham, D. W., \& Aguilar, L. A. 1996, AJ, 112, 668
Carney, B. W., Storm, J., \& Jones, R. V. 1992, ApJ, 386, 663 (CSJ92)

Carretta, E., \& Bragaglia, A. 1998, A\&A, 329, 937

Carretta, E., Cacciari, C., Ferraro, F. R., Fusi Pecci, F., \& Tessicini, G. 1998, MNRAS, 298, 1005

Carretta, E., \& Gratton, R. G. 1997, A\&AS, 121, 95

Cassisi, S., Degl'Innocenti, S., \& Salaris, M. 1997, MNRAS, 290, 515

Cassisi, S., \& Salaris, M. 1997, MNRAS, 285, 593

Castellani, V., Maceroni, C., \& Tosi, M. 1983, A\&A, 128, 64

Castellani, V., \& Quarta, M. L. 1987, A\&AS, 71, 1

Castellani, V., \& Tornambé, A. 1981, A\&A, 96, 207

Catelan, M. 1998, ApJ, 495, L81

Catelan, M., Borissova, J., Sweigart, A. V., \& Spassova, N. 1998, ApJ, 494, 265

Chaboyer, B., Demarque, P., Kernan, P. J., \& Krauss, L. M. 1998, ApJ, 494, 96 
Chiosi, C., Bertelli, G., \& Bressan, A. 1992, ARA\&A, 30, 235

Clement, C. M., \& Shelton, I. 1999, ApJ, 515, L85

Da Costa, G. S., \& Armandroff, T. E. 1995, AJ, 109, 2533

Dixon, W. V. D., Davidsen, A. F., Dorman, B., \& Ferguson, H. C. 1996, AJ, 111,1936

Dorman, B. 1992, ApJS, 81, 221

Ferraro, F. R., Carretta, E., Corsi, C. E., Fusi Pecci, F., Cacciari, C., Buonanno, R., Paltrinieri, B., \& Hamilton, D. 1997, A\&A, 320, 757

Frenk, C. S., \& White, S. D. M. 1980, MNRAS, 199, 565

Fusi Pecci, F., et al. 1996, AJ, 112, 1461

Fusi Pecci, F., Ferraro, F. R., Crocker, D. A., Rood, R. T., \& Buonanno, R. 1990, A\&A, 238, 95

Goldsmith, C. G. 1993, in New Perspectives on Stellar Evolution, ed. J. M. Nemec \& J. M. Mattews (Cambridge: Cambridge Univ. Press), 358

Gonzalez, G., \& Wallerstein, G. 1998, AJ, 116, 765

Hazen, M. L., \& Nemec, J. M. 1992, AJ, 104, 111

Isobe, T., Feigelson, E. D., Akritas, M. G., \& Babu, G. J. 1990, ApJ, 364, 104

Kinman, T. D., Kraft, R. P., Friel, E., \& Suntzeff, N. B. 1985, AJ, 90, 95

Kinman, T. D., Mahaffey, C. T., \& Wirtanen, C. A. 1982, AJ, 87, 314

Kinman, T. D., Suntzeff, N. B., \& Kraft, R. P. 1994, AJ, 108, 1722

Kinman, T. D., Wong-Swanson, B., Wenz, M., \& Harlan, E. A. 1984, AJ, 89,1200

Kraft, R. P., Sneden, C., Langer, G. E., \& Prosser, C. F. 1992, AJ, 104, 645

Kraft, R. P., Sneden, C., Smith, G., Shetrone, M. D., \& Fulbright, J. 1998, AJ, 115, 1500

Layden, A. C. 1996, in ASP Conf. Ser. 92, Formation of the Galactic Halo...Inside and Out, ed. H. Morrison \& A. Sarajedini (San Francisco: ASP), 141

Lee, Y.-W. 1990, ApJ, 363, 159

. 1991, ApJ, 367, 524

Lee, J.-W., \& Carney, B. W. 1999, AJ, 117, 2868 (LC99)

Lee, Y.-W., \& Demarque, P. 1990, ApJS, 73, 709

Lee, Y.-W., Demarque, P., \& Zinn, R. 1990, ApJ, 350, 155 (LDZ90) 1994, ApJ, 423, 248 (LDZ94)

Liller, M. H., \& Lichten, S. M. 1978, AJ, 83, 1070

Majewski, S. R. 1992, ApJS, 78, 87

Nemec, J. M. 1985, AJ, 90, 204

Nemec, J. M., Wehlau, A., \& Mendes de Oliveira, C. 1988, AJ, 96, 528

Press, W. H., Teukolsky, S. A., Vetterling, W. T., \& Flannery, B. P. 1992, Numerical Recipes (Cambridge: Cambridge Univ. Press)

Preston, G. W. 1959, ApJ, 130, 507

Preston, G. W., Shectman, S. A., \& Beers, T. C. 1991, ApJ, 375, 121
Ratnatuga, K. U., \& Bahcall, J. N. 1985, ApJS, 59, 63

Renzini, A., \& Fusi Pecci, F. 1988, ARA\&A, 26, 199

Rodgers, A. W., \& Paltoglou, G. 1984, ApJ, 283, L5

Rood, R. T. 1990, in ASP Conf. Ser. 11, Confrontation Between Stellar Pulsation and Evolution, ed. C. Cacciari \& G. Clementini (San Francisco: ASP), 11

Saha, A. 1984, ApJ, 283, 580

Saha, A., Monet, D. G. \& Seitzer, P. 1986, AJ, 92, 302

Saha, A., \& Oke, J. B. 1984, ApJ, 285, 688

Sandage, A. 1981, ApJ, 248, 161

. 1982, ApJ, 252, 553

1990a, ApJ, 350, 603 1990b, ApJ, 350, 631 .1993, AJ, 106, 687

Sandage, A., Katem, B., \& Sandage, M. 1981, ApJS, 46, 41

Sandage, A., \& Smith, L. L. 1966, ApJ, 144, 886

Sandage, A., \& Wallerstein, G. 1960, ApJ, 131, 598

Sarajedini, A., Chaboyer, B., \& Demarque, P. 1997, PASP, 109, 1321

Searle, L., \& Zinn, R. 1978, ApJ, 225, 357

Smith, H. A. 1995, RR Lyrae Stars (Cambridge: Cambridge Univ. Press)

Stetson, P. B., VandenBerg, D. A., \& Bolte, M. 1996, PASP, 108, 560

Suntzeff, N. B., Kinman, T. D., \& Kraft, R. D. 1991, ApJ, 367, 528

Suntzeff, N. B., Kraft, R. P.. \& Kinman, T. D. 1994, ApJS, 93, 271 (SKK94)

Sweigart, A. V. 1997, ApJ, 474, L23

Sweigart, A. V., Renzini, A., \& Tornambè, A. 1987, ApJ, 312, 762

van Agt, S. 1973, in Variable Stars in Globular Clusters, ed. J. D. Fernie (Dordrecht: Reidel), 35

van Albada, T. S., \& Baker, N. 1971, ApJ, 169, 311 1973, ApJ, 185, 477

VandenBerg, D. A., Bolte, M., \& Stetson, P. B. 1990, AJ, 100, 445

van den Bergh, S. 1993a, MNRAS, 262, 588 .1993b, AJ, 105, 971

Wehlau, A., Nemec, J., Hanlan, P., \& Rich, R. M. 1992, AJ, 103, 1583

Wehlau, A., Slawson, R. W., \& Nemec, J. M. 1999, AJ, 117, 286

Wilhelm, R., Beers, T. C., Kriessler, J. R., Pier, J. R., Sommer-Larsen, J., \& Layden, A. C. 1996, in ASP Conf. Ser. 92, Formation of the Galactic Halo...Inside and Out, ed. H. Morrison \& A. Sarajedini (San Francisco: ASP), 171

Yi, S., Demarque, P., \& Kim, Y.-C. 1997, ApJ, 482, 677

Zinn, R. 1985, ApJ, 293, 424

1993, in ASP Conf. Ser. 48, The Globular Cluster-Galaxy Connection, ed. G. H. Smith \& J. P. Brodie (San Francisco: ASP), 38

Zinn, R., \& West, M. J. 1984, ApJS, 55, 45 